\title{
A RICCI-TYPE FLOW ON GLOBALLY NULL MANIFOLDS AND ITS GRADIENT ESTIMATES
}

\author{
MOHAMED H. A. HAMED, FORTUNÉ MASSAMBA, AND SAMUEL SSEKAJJA
}

\begin{abstract}
Locally, a screen integrable globally null manifold $M$ splits through a Riemannian leaf $M^{\prime}$ of its screen distribution and a null curve $\mathcal{C}$ tangent to its radical distribution. The leaf $M^{\prime}$ carries a lot of geometric information about $M$ and, in fact, forms a basis for the study of expanding and nonexpanding horizons in black hole theory. In the present paper, we introduce a degenerate Ricci-type flow in $M^{\prime}$ via the intrinsic Ricci tensor of $M$. Several new gradient estimates regarding the flow are proved.
\end{abstract}

\section{INTRODUCTION}

Let $M$ be a compact $m$-dimensional Riemannian manifold on which a one parameter family of Riemannian metrics $g(t), t \in[0, T], T<T_{\epsilon}$, where $T_{\epsilon}$ is the time where there is (possibly) a blow-up of the curvature, is defined. We say that $(M, g(t))$ is a solution to the Ricci flow if it is evolving by the following non-linear weakly parabolic partial differential equation (see [1]):

$$
\partial_{t} g_{i j}(x, t)=-2 \operatorname{Ric}_{i j}(x, t), \quad(x, t) \in M \times[0, T],
$$

with $g_{i j}(x, 0)=g_{i j}(x)$, where $\operatorname{Ric}_{i j}(x, t)$ is the Ricci curvature tensor of the evolving metric $g_{i j}(x, t)$. This evolution system was initially introduced by Hamilton in [12]. The evolution equation for the metric tensor implies the evolution equation for the curvature tensor $R$ in the form $\partial_{t} R=\Delta R+Q$, where $\Delta$ denotes the Laplacian operator on $M$ and $Q$ is a quadratic expression of the curvatures. In particular, the scalar curvature $\widetilde{R}$ satisfies $\partial_{t} \widetilde{R}=\Delta \widetilde{R}+2|\widetilde{\mathrm{Ric}}|^{2}$, so by the maximum principle its minimum is non-decreasing along the flow. By developing a maximum principle for tensors, Hamilton 12 proved that the Ricci flow preserves the positivity of the Ricci tensor in dimension three and of the curvature operator in all dimensions; moreover, the eigenvalues of the Ricci tensor in dimension three and of the curvature operator in dimension four are getting pinched pointwisely as the curvature gets

2020 Mathematics Subject Classification. Primary 53E10; Secondary 53C40, 53C50.

Key words and phrases. Null manifold, Screen integrable distribution, Ricci-type flow.

Mohamed H. A. Hamed and Samuel Ssekajja would like to thank the Simons Foundation through the RGSM-Project for financial support. This work is based on research supported wholly/in part by the National Research Foundation of South Africa (grant numbers 95931 and 106072). 
larger. In [22, Perelman used Ricci flow and its surgery to prove the Poincaré Conjecture. In the papers [1] and [11] the authors used Ricci flow coupled to heatlike equations to study some gradient estimates. For more information about the classical Ricci flow, see [1, 2, 6, 12, 22, 23, and references therein.

When the underlying manifold $M$ is null (sometimes called degenerate or lightlike), one may not define, in the usual way, the Ricci flow associated to the degenerate metric $g$ on $M$. In fact, it is well known (see [7) that, in general, there is no Ricci tensor on $M$ via the null metric $g$. When $M$ is embedded into a semiRiemannian manifold $\bar{M}$ as a null hypersurface, special classes of $M$ do exist with induced symmetric Ricci tensors. For instance, in 10 the authors show that null hypersurfaces $\mathcal{C}[M]^{0}$ of genus zero exhibit an induced Ricci tensor. As the Ricci flow is an intrinsic geometric flow, one need not know much about the ambient space in which $M$ is embedded. In [14, Kupeli studies null manifolds using a factor bundle approach and proves the existence of many geometric objects on $M$ by taking the assumption that $M$ is stationary, i.e., the normal bundle of $M$ is a Killing distribution, which ensures a Levi-Civita connection on $M$. In fact, a stationary $M$ is the same as a globally null manifold studied by Duggal in 8. A screen integrable globally null manifold $M$ is locally a product manifold of a leaf $M^{\prime}$ of its screen distribution and a null curve $\mathcal{C}$ tangent to the normal bundle of $M$. The leaves $M^{\prime}$ are fundamental in studying expanding and non-expanding black hole horizons in mathematical physics; see for instance [14, 21] and references therein. As the intrinsic Ricci tensor of $M$ is symmetric on $M^{\prime}$, we introduce a degenerate Ricci-type flow on $M$ using such a Ricci tensor and investigate its properties in terms of the associated gradient estimates.

The theory of null submanifolds of a semi-Riemannian manifold is one of the most important topics of differential geometry. More precisely, null hypersurfaces appear in general relativity as models of different types of black hole horizons [7, 10, 21. The study of non-degenerate submanifolds of semi-Riemannian manifolds has many similarities with that of Riemannian submanifolds. However, in case the induced metric on the submanifold is degenerate, the study becomes more difficult and is strikingly different from the study of non-degenerate submanifolds [7]. Some of the pioneering works on null geometry is due to Duggal-Bejancu [7], DuggalSahin [10, and Kupeli [14]. Such works motivated many other researchers to pursue the study of null submanifolds, for example [6, 7, 9, 14, 16, 17, 18, 19, 20] and many more references therein. The rest of the paper is organized as follows. In Section 2 we review the basics on globally null manifolds. In Section 3 we define a degenerate Ricci-type flow evolution on a globally null manifold and give some examples. In Sections 4 and 5 we develop several gradient estimates for the degenerate Ricci-type flow.

\section{Globally null manifolds}

We recall the basic concepts on globally null manifolds (for more details, see [8] and references therein). Let $(M, g)$ be a real $m$-dimensional smooth manifold where $g$ is a symmetric tensor field of type $(0,2)$. We assume that $M$ is paracompact. For 
$x \in M$, the radical or null space of $T_{x} M$ is the subspace, denoted by $\operatorname{Rad} T_{x} M$, defined by (see [13] for more details)

$$
\operatorname{Rad} T_{x} M=\left\{E_{x} \in \operatorname{Rad} T_{x} M: g\left(E_{x}, X\right)=0, X \in T_{x} M\right\} .
$$

The dimension, say $r$, of $\operatorname{Rad} T_{x} M$ is called the nullity degree of $g . \operatorname{Rad} T_{x} M$ is called the radical distribution of rank $r$ on $M$. Clearly, $g$ is degenerate or nondegenerate on $M$ if and only if $r>0$ or $r=0$, respectively. We say that $(M, g)$ is a null manifold if $0<r \leq m$.

In this paper, we assume that $0<r<m$. Consider a complementary distribution $S(T M)$ to $\operatorname{Rad} T M$ in $T M$. We call $S(T M)$ a screen distribution on $M$, and its existence is ensured by the paracompactness of $M$. It is easy to see that $S(T M)$ is semi-Riemannian. Therefore, we have the decomposition

$$
T M=S(T M) \oplus \operatorname{Rad} T M .
$$

The associated quadratic form of $g$ is a mapping $h: T_{x} M \rightarrow \mathbb{R}$ given by $h(X)=$ $g(X, X)$, for any $X \in T_{x} M$. In general, $h$ is of type $(p, q, r)$, where $p+q+r=m$ and $q$ is the index of $g$ on $T_{x} M$. We use the following range of indices: $I, J \in$ $\{1, \ldots, q\}, A, B \in\{q+1, \ldots, q+p\}, \alpha, \beta \in\{1, \ldots, r\}$, and $a, b \in\{r+1, \ldots, m\}$, $i, j \in\{1, \ldots, m\}$.

Throughout the paper we consider $\Gamma(\Xi)$ to be a set of smooth sections of the vector bundle $\Xi$.

Using a well-known result from linear algebra, we have the following canonical form for $h$ (with respect to a local basis of $T_{x} M$ ):

$$
h=-\sum_{I=1}^{q}\left(\omega^{I}\right)^{2}+\sum_{A=q+1}^{p+q}\left(\omega^{A}\right)^{2},
$$

where $\omega^{1}, \ldots, \omega^{p+q}$ are linearly independent local differential 1-forms on $M$. With respect to a local coordinate system $\left(x^{i}\right)$, the above relation leads to

$$
\begin{aligned}
h & =-\sum_{I=1}^{q}\left(\omega^{I}\right)^{2}+\sum_{A=q+1}^{q+p}\left(\omega^{A}\right)^{2}, \quad h=g_{i j} d x^{i} d x^{j}, \quad \operatorname{rank}\left|g_{i j}\right|=p+q<m, \\
g_{i j} & =g\left(\partial_{i}, \partial_{j}\right)=-\sum_{I=1}^{q} \omega_{i}^{I} \omega_{j}^{I}+\sum_{A=q+1}^{q+p} \omega_{i}^{A} \omega_{j}^{A},
\end{aligned}
$$

since $\omega^{I}=\omega_{i}^{I} d x^{i}$ and $\omega^{A}=\omega_{i}^{A} d x^{i}$.

Suppose that $\operatorname{Rad} T M$ is an integrable distribution. Then it follows from the Frobenius theorem that the leaves of $\operatorname{Rad} T M$ determine a foliation on $M$ of dimension $r$, that is, $M$ is a disjoint union of connected subsets $\left\{L_{t}\right\}$ and at each point $x \in M, M$ has a coordinate system $\left(\mathcal{U}, x^{i}\right)$, where $i \in\{1, \ldots, m\}$ and $L_{t} \cap \mathcal{U}$ is locally given by the equation $x^{a}=c^{a}, a \in\{r+1, \ldots, m\}$ for real constants $c^{a}$, and $\left(x^{\alpha}\right), \alpha \in\{1, \ldots, r\}$, are local coordinates of a leaf $L$ of $\operatorname{Rad} T M$. Consider another coordinate system $\left(\overline{\mathcal{U}}, \bar{x}^{\alpha}\right)$ on $M$. The transformation of coordinates on $M$, endowed with an integrable distribution, has the special form $0=d \bar{x}^{a}=\frac{\partial \bar{x}^{a}}{\partial \bar{x}^{b}} d x^{b}+\frac{\partial \bar{x}^{a}}{\partial \bar{x}^{\alpha}} d x^{\alpha}=\frac{\partial \bar{x}^{a}}{\partial \bar{x}^{\alpha}} d x^{\alpha}$, which implies $\frac{\partial \bar{x}^{a}}{\partial \bar{x}^{\alpha}}=0$, for all 
$a \in\{r+1, \ldots, m\}$ and $\alpha \in\{1, \ldots, r\}$. Hence the transformation of coordinates on $M$ is given by

$$
\bar{x}^{\alpha}=\bar{x}^{\alpha}\left(x^{1}, \ldots, x^{r}\right), \quad \bar{x}^{a}=\bar{x}^{a}\left(x^{r+1}, \ldots, x^{m}\right) .
$$

As $g$ is degenerate on $T M$, by using (2.1) and the canonical form for $h$ we obtain $g_{\alpha \beta}=g_{\alpha a}=g_{a \alpha}=0$. Thus, the matrix of $g$ with respect to the natural frame $\left\{\partial_{i}\right\}$ becomes

$$
\left(g_{i j}\right)=\left(\begin{array}{cc}
O_{r, r} & O_{r, m-r} \\
O_{m-r, r} & g_{a b}\left(x^{1}, \ldots, x^{m}\right)
\end{array}\right) .
$$

By the coordinates in 2.2 , one can show that

$$
\partial_{\alpha} g_{a b}=0, \quad \text { for all } a, b \in\{r+1, \ldots, m\}, \alpha \in\{1, \ldots, r\},
$$

holds for any other coordinate system adapted to the foliation induced by $\operatorname{Rad} T M$. We will suppose, therefore, that 2.3 holds. Also, one can show that the screen distribution $S(T M)$ is invariant with respect to the transformation in 2.2 .

Next, we assume that $r=1$. Thus, the 1-dimensional nullity distribution $\operatorname{Rad} T M$ is integrable. The following result was established in 8 .

Theorem $2.1([8])$. Let $(M, g)$ be an m-dimensional null manifold, with $\operatorname{Rad} T M$ of rank 1. Then, there exists a Levi-Civita metric connection $\nabla$ on $M$ with respect to the degenerate metric tensor $g$.

Let $C$ be a null curve in an $m$-dimensional null manifold $(M, g)$, with $m>1$ and locally given by $x^{i}=x^{i}(t), t \in I \subset \mathbb{R}, i \in\{1, \ldots, m\}$, for a coordinate neighborhood $\mathcal{U}$ on $C$. Then, the tangent vector field $\frac{d}{d t}=\left(\frac{d x^{1}}{d t}, \ldots, \frac{d x^{m}}{d t}\right)$ on $\mathcal{U}$ satisfies $g\left(\frac{d}{d t}, \frac{d}{d t}\right)=0$, i.e., $g_{i j} \frac{d x^{i}}{d t} \frac{d x^{j}}{d t}=0$, where $g_{i j}=g\left(\partial_{i}, \partial_{j}\right)$ and $i, j \in\{1, \ldots, m\}$. Denote by $T C$ the tangent bundle of $C$, which is a vector subbundle of $T M$ and has rank 1. It is easy to see that $T C^{\perp}=\{V \in T M: g(V, E)=0\}=T M$, where $E$ is a null vector field tangent over $C$. Suppose that $\operatorname{Rad} T M$ is of $\operatorname{rank} 1$. We consider a class of null curves such that $\operatorname{Rad} T M=T C$ and both are generated by a null vector field $E$ tangent over $C$. Let $S(T M)$ be the complementary screen distribution to $\operatorname{Rad} T M$. Then, we have

$$
T M=\operatorname{Rad} T M \perp S(T M)=T C \perp S(T M),
$$

where $\perp$ means the orthogonal direct sum. It follows that $S(T M)$ is the transversal vector bundle of $C$ in $T M$. Suppose that $S(T M)$ is Riemannian and $m=3$; we obtain the differential equations

$$
\nabla_{E} E=\widetilde{h} E, \quad \nabla_{E} W_{1}=-k_{1} E+k_{3} W_{2}, \quad \nabla_{E} W_{2}=-k_{2} E-k_{3} W_{1},
$$

where $\widetilde{h}$ and $\left\{k_{1}, k_{2}, k_{3}\right\}$ are smooth functions on $\mathcal{U}$ and $\left\{W_{1}, W_{2}\right\}$ is an orthonormal basis of $\Gamma(S(T M)) \mathcal{U}$. We call $F=\left\{\frac{d}{d t}=E, W_{1}, W_{2}\right\}$ a Frénet frame on $M$ along $C$ with respect to the screen distribution $S(T M)$. The functions $\left\{k_{1}, k_{2}, k_{3}\right\}$ and the differential equations (2.4) are called curvature functions of $C$ and Frénet equations for $F$, respectively. The result can be generalized for higher dimensions. It is important to mention that for any $m$, the first Frénet equation for $F$ remains the same. Now we show that it is always possible to find a parameter on $C$ such that 
$\widetilde{h}=0$, using the same screen distribution $S(T M)$. Consider another coordinate neighborhood $\mathcal{U}^{*}$ and its Frénet frame $F^{*}$, with $\mathcal{U} \cap \mathcal{U}^{*} \neq \emptyset$. Then, $\frac{d}{d t^{*}}=\frac{d t}{d t^{*}} \frac{d}{d t}$.

Writing the first Frénet equation in 2.4 for both $F$ and $F^{*}$ and using the above transformation, we obtain $\frac{d^{2} t}{d t^{* 2}}+\widetilde{h}\left(\frac{d t}{d t^{*}}\right)^{2}=\widetilde{h}^{*} \frac{d t}{d t^{*}}$. Consider the differential equation $\frac{d^{2} t}{d t^{* 2}}-\widetilde{h}^{*} \frac{d t}{d t^{*}}=0$, whose general solution comes from

$$
t=a \int_{t_{0}}^{t^{*}} \exp \left(\int_{s_{0}}^{s} \widetilde{h}^{*}\left(t^{*}\right) d t^{*}\right) d s+b, \quad a, b \in \mathbb{R} .
$$

It follows that a solution of $(2.5)$, with $a \neq 0$, may be taken as a special parameter on $C$ such that $\widetilde{h}=0$. Denote such a parameter by $p=\frac{t-b}{a}$, where we call $t$ the general parameter as given in (2.5), and $p$ a distinguished parameter of $C$. Then, the first Frénet equation is given by $\nabla_{\frac{d}{d p}} \frac{d}{d p}=0$ and, therefore, $C$ is a null geodesic of $M$ with respect to the distinguished parameter $p$. Since the first Frénet equation is the same for any $m$, the following holds.

Theorem 2.2 ([8]). Let $C$ be a null curve of a null manifold $M$, with $\operatorname{Rad} T M$ of rank 1 and Riemannian screen distribution. Then, there exists a distinguished parameter $p$ with respect to which $C$ is a null geodesic of $M$.

In view of the above theorem, the author in 8 defined globally null manifolds as follows.

A null manifold $(M, g)$ is said to be a globally null manifold if it admits a single global null vector field and a complete Riemannian hypersurface.

As a consequence, the following characterization holds on globally null manifolds.

Theorem $2.3([\underline{8})$. Let $(M, g)$ be a globally null manifold. Then, the following assertions are equivalent:

(1) The screen distribution $S(T M)$ is integrable.

(2) $M=M^{\prime} \times C^{\prime}$ is a global product manifold, where $M^{\prime}$ is a leaf of $S(T M)$ and $C^{\prime}$ is a 1-dimensional integral manifold of a global null curve $C$ in $M$.

(3) $S(T M)$ is parallel with respect to the metric connection $\nabla$ on $M$.

As an example, we have the following.

Example 2.4. Let $\left(\mathbb{R}_{1}^{4}, \bar{g}\right)$ be the Minkowski spacetime with metric $d s^{2}=-d t^{2}+$ $d r^{2}+r^{2}\left(d \theta^{2}+\sin ^{2} \theta d \phi^{2}\right)$, for a spherical coordinate system $(t, r, \theta, \phi)$, which is nonsingular if we restrict $0<r<\infty, 0<\theta<\pi, 0<\phi<2 \pi$. It is well known that Minkowski spacetime is globally hyperbolic. Take two null coordinates $u=t+r$ and $v=t-r(u>v)$. Then, we have

$$
d s^{2}=-d u d v+\frac{1}{4}(u-v)^{2}\left(d \theta^{2}+\sin ^{2} \theta d \phi^{2}\right), \quad-\infty<u, v<\infty .
$$

The absence of the terms $d u^{2}$ and $d v^{2}$ in 2.6 implies that the two hypersurfaces $\{v=$ constant $\},\{u=$ constant $\}$ are null. Denote one of these null hypersurfaces by $(M, g)$, where $g$ is the induced degenerate metric tensor of $\bar{g}$. A leaf of the 2-dimensional screen distribution $S(T M)$ is topologically a 2-sphere with complete Riemannian metric $d \Omega^{2}=r^{2}\left(d \theta^{2}+\sin ^{2} \theta d \phi^{2}\right)$, which is the intersection of the 
two hypersurfaces. Since by definition a spacetime admits a global timelike vector field, it follows that both its null hypersurfaces admit a single global null vector field. Thus, there exists a pair of globally null manifolds, as null hypersurfaces of a Minkowski spacetime.

Remark 2.5. Globally null manifolds were also studied by Kupeli [14, under the name of stationary singular semi-Riemannian manifolds.

\section{A DEgenerate RicCi-TyPe Flow}

Let $(M, g)$ be a null manifold and $S(T M)$ be its screen distribution. A globally null manifold $(M, g)$ is said to be a screen integrable globally null manifold if its screen distribution $S(T M)$ is integrable.

Let $(M, g)$ be a screen integrable globally null manifold and $\left(M^{\prime}, g^{\prime}\right)$ be a leaf of $S(T M)$ such that $g^{\prime}=\left.g\right|_{M^{\prime}}$, immersed in $M$ as a non-degenerate submanifold. Denote by $P$ the projection morphism of $T M$ into $S(T M)$. Let $R$ denote the curvature tensor of $M$ with respect to the metric connection $\nabla$ on $M$. If $R^{\prime}$ is the curvature tensor of $M^{\prime}$ with respect to the connection $\nabla^{\prime}$ on $M^{\prime}$, then, in view of [14, p. 48], we have

$$
R^{\prime}(X, Y) Z^{\prime}=P R(X, Y) Z
$$

for all $X, Y, Z \in \Gamma(T M)$ with $Z^{\prime}=P Z$. The intrinsic curvature tensor $\bar{R}$ of $\left(M^{\prime}, g^{\prime}\right)$ is then given by $\bar{R}\left(X^{\prime}, Y^{\prime}\right) Z^{\prime}=R^{\prime}(X, Y) Z^{\prime}$, where $X^{\prime}=P X, Y^{\prime}=P Y$, and $Z^{\prime}=P Z$, for all $X, Y, Z \in \Gamma(T M)$. $\bar{R}$ satisfies the usual properties of Riemannian curvature tensors (see [14, Theorem 3.2.10]). Note that $\bar{R}$ is tensorial with respect to its entries, hence for any $x \in M,\left(\bar{R}\left(X^{\prime}, Y^{\prime}\right) Z^{\prime}\right)_{x}$ depends on the values of $X^{\prime}, Y^{\prime}, Z^{\prime} \in T_{x} M^{\prime}$. Moreover, the associated curvature-like tensor of type $(0,4)$ is given by

$$
\bar{R}\left(X^{\prime}, Y^{\prime}, Z^{\prime}, W^{\prime}\right)=g^{\prime}\left(\bar{R}\left(X^{\prime}, Y^{\prime}\right) Z^{\prime}, W^{\prime}\right),
$$

where $X^{\prime}, Y^{\prime}, Z^{\prime}, W^{\prime} \in \Gamma\left(T_{x} M^{\prime}\right)$.

Let $(M, g)$ be a screen integrable global null submanifold. Then, the Ricci tensor $\operatorname{Ric}^{\prime}$ of $M^{\prime}$ is defined by $\operatorname{Ric}^{\prime}\left(X^{\prime}, Y^{\prime}\right)=\operatorname{trace}\left\{Z^{\prime} \rightarrow \bar{R}\left(Z^{\prime}, X^{\prime}\right) Y^{\prime}\right\}$, for all $X^{\prime}, Y^{\prime}, Z^{\prime} \in \Gamma\left(T_{x} M^{\prime}\right)$. The corresponding scalar curvature $S c a l^{\prime} \in C^{\infty}\left(M^{\prime}\right)$ of $\left(M^{\prime}, g^{\prime}\right)$ is defined by $S c a l^{\prime}=\operatorname{trace}\left(\operatorname{Ric}^{\prime}\right)$. For a function $f \in C^{\infty}(M)$, we will denote by $\bar{f}=\left.f\right|_{M^{\prime}}$ its restriction to $M^{\prime}$. Recall that for a smooth function $\bar{f}$, we define a symmetric 2-tensor $\bar{\nabla}^{2} \bar{f}$ by $\bar{\nabla}^{2} \bar{f}\left(X^{\prime}, Y^{\prime}\right)=X^{\prime} Y^{\prime} \bar{f}-\left(\nabla_{X^{\prime}} Y^{\prime}\right) \bar{f}=$ $\operatorname{Hess}(\bar{f})\left(X^{\prime}, Y^{\prime}\right)$, from which we define the Laplacian $\Delta \bar{f}$ of $\bar{f}$ as $\Delta \bar{f}=\operatorname{trace} \bar{\nabla}^{2} \bar{f}=$ $g^{\prime a b}\left(\bar{\nabla}^{2} \bar{f}\right)_{a b}$. Notice that $\underline{\underline{f}}=\operatorname{div}(\bar{\nabla} \bar{f})$, where $\bar{\nabla} \bar{f}$ denotes the gradient of $\bar{f}$ and $\operatorname{div}(\cdot)$ denotes the divergence operator on $M^{\prime}$.

For more details of curvature properties on globally null manifolds, see Kupeli's book [14]. In view of the above background, we define a degenerate Ricci-type flow on $(M, g)$ as follows.

Definition 3.1. Let $(M, g)$ be a screen integrable globally null manifold, and let $\left(M^{\prime}, g^{\prime}=\left.g\right|_{M^{\prime}}\right)$ be a leaf of $S(T M)$. A degenerate Ricci-type flow of $(M, g)$ is 
a family $\left\{g^{\prime}(t)\right\}_{t \in I}$ of Riemannian metrics on $\left(M^{\prime}, g^{\prime}\right)$, parametrized by a time interval $I \subset \mathbb{R}$ and evolving by

$$
\partial_{t} g^{\prime}(t)=-2 \operatorname{Ric}^{\prime}\left(g^{\prime}(t)\right)
$$

In harmonic local coordinates around a point $x \in M$, the Ricci tensor at $x$ is $\operatorname{Ric}_{a b}^{\prime}(x)=-\frac{1}{2} \Delta g_{a b}^{\prime}(x)$. Thus, a degenerate Ricci-type flow resembles a heat flow evolution.

Next, we will give some examples of degenerate Ricci flows.

Example 3.2. Let $(M, g)$ be a globally null manifold and let $\phi: M \rightarrow \mathbb{R}_{1}^{n+2}$ be an isometric immersion of $M$ into $\mathbb{R}_{1}^{n+2}$ as a null hypersurface. Then, by the definition of globally null manifolds, $M$ is embedded in $\mathbb{R}_{1}^{n+2}$ as totally geodesic hypersurface. As $\mathbb{R}_{1}^{n+2}$ is flat, we notice that $M$ is flat as well (see [7] for more details). Consequently, the intrinsic curvature $\bar{R}$ vanishes and each leaf $M^{\prime}$ is flat. Thus, the flat metric on $M^{\prime}$ has zero Ricci curvature, so it does not evolve at all under the degenerate Ricci-type flow.

Example 3.3. Consider the globally null manifold $(M, g)$ of Example 2.4 Each leaf $M^{\prime}$ of $S(T M)$ is a 2-dimensional sphere of radius $r$; the metric is given by $g^{\prime}=r^{2} g^{\prime \prime}$, where $g^{\prime \prime}=d \theta^{2}+\sin ^{2} \theta d \phi^{2}$ is the metric on the unit sphere. The sectional curvatures are all $1 / r^{2}$. Thus for any unit vector field $X^{\prime} \in \Gamma\left(T M^{\prime}\right)$, we have $\operatorname{Ric}^{\prime}\left(X^{\prime}, X^{\prime}\right)=1 / r^{2}$. Therefore, $\operatorname{Ric}^{\prime}=\frac{1}{r^{2}} g^{\prime}=g^{\prime \prime}$, so the degenerate Riccitype flow equation becomes an ordinary differential equation $\partial_{t} g^{\prime}=-2 \mathrm{Ric}^{\prime}$, from which we obtain $\frac{d\left(r^{2}\right)}{d t}=-2$. Solving this ODE gives $r(t)=\sqrt{R_{0}^{2}-2 t}$, where $R_{0}$ is the initial radius of the sphere. Notice that the degenerate Ricci-type flow of $(M, g)$ will become singular at $t=(1 / 2) R_{0}^{2}$. At this time, the leaf $M^{\prime}$ has collapsed to a point.

The existence and uniqueness of degenerate Ricci-type flows can be established in the same way as in the classical Ricci flow, as well as the associated geometric evolution equations for the induced objects associated to curvature (see [2, p. 90] for more details).

As each leaf $M^{\prime}$ is Riemannian, we can define the distance function on $M^{\prime}$ in a natural way. For a point $p \in M^{\prime}$, define $d(x, p)$ for all $x \in M^{\prime}$, where $d(\cdot, \cdot)$ is the geodesic distance. It is important to note that $d$ is only Lipschitz continuous, that is, everywhere continuous except at the cut locus of $p$ and on the point where $x$ coincides with $p$. One can easily see that $|\bar{\nabla} d|=g^{\prime a b} \partial_{a} d \partial_{b} d=1$ on $M^{\prime} /\{\{p\} \cup$ $\operatorname{cut}(p)\}$. Let $d(x, y, t)$ be the geodesic distance between $x$ and $y$ with respect to the Riemannaian metric $g^{\prime}(t)$; we define a smooth cut-off function $\varphi(x, t)$ with support in the geodesic cube

$$
\mathcal{Q}_{2 \rho, T}:=\left\{(x, y) \in M^{\prime} \times(0, T]: d(x, y, t) \leq 2 \rho\right\},
$$

for any $C^{2}$-function $\psi$ on $[0, \infty)$ with $\psi(s)=1$ for $s \in[0,1]$ and $\psi(s)=0$ for $s \in[2,+\infty]$ (see [1] for more details). Furthermore, $\psi^{\prime}(s) \leq 0, \psi^{\prime \prime}(s) \geq-c_{1}$, and $\frac{\left|\psi^{\prime}\right|^{2}}{\psi} \leq c_{2}$, where $c_{1}, c_{2}$ are constants such that $\varphi(x, t)=\psi(d(x, p, t) / \rho)$ and $\left.\varphi\right|_{\mathcal{Q}_{2 \rho, T}}=1$. 
As in 1], let $M^{\prime}$ be a complete $n$-dimensional leaf of the integrable screen distribution $S(T M)$ of a null manifold $(M, g)$ whose Ricci curvature is bounded from below by $\operatorname{Ric}^{\prime} \geq(n-1) k$, for some constant $k \in \mathbb{R}$. Then the Laplacian of the distance function satisfies

$$
\underline{\Delta} d(x, p)= \begin{cases}(n-1) \sqrt{k} \cot (\sqrt{k} \rho), & k>0, \\ (n-1) \rho^{-1}, & k=0, \\ (n-1) \sqrt{|k|} \operatorname{coth}(\sqrt{k} \rho), & k<0 .\end{cases}
$$

Throughout, we will impose boundedness conditions on the Ricci curvature of the metric. Note that when the metric evolves by the degenerate Ricci-type flow, boundedness and sign assumptions are preserved as long as the flow exists. So also the metrics are uniformly equivalent. If $-\rho_{1} g^{\prime} \leq \operatorname{Ric}^{\prime} \leq \rho_{2} g^{\prime}$, where $g^{\prime}(t)$, $t \in(0, T]$, is a degenerate Ricci flow, then

$$
e^{-\rho_{1} T} g^{\prime}(0) \leq g^{\prime}(t) \leq e^{-\rho_{2} T} g^{\prime}(0) .
$$

\section{Some Gradient estimates}

In this section, we discuss the localized version of gradient estimates on the heat equation perturbed with curvature operator under both forward and backward degenerate Ricci-type flow. The estimate under backward action of degenerate Ricci-type flow is related to the local monotonicity for heat kernels and mean value theorem of Ecker, Knopf, Ni, and Topping in [11]. They worked in general geometric flow, and we follow their approach.

Let $(M, g)$ be a screen integrable globally null manifold and $\left(M^{\prime}, g^{\prime}=\left.g\right|_{M^{\prime}}\right)$ be a leaf of the screen distribution $S(T M)$. We consider the conjugate heat equation coupled to the backward and forward degenerate Ricci-type flow, respectively, as follows:

$$
\left\{\begin{array} { l } 
{ ( \partial _ { t } - \underline { \Delta } + S c a l ^ { \prime } ) u ( x , t ) = 0 , } \\
{ \partial _ { t } g ^ { \prime } ( x , t ) = 2 \operatorname { R i c } ^ { \prime } ( x , t ) , }
\end{array} \quad \text { and } \quad \left\{\begin{array}{l}
\left(\partial_{t}-\underline{\Delta}+S c a l^{\prime}\right) u(x, t)=0 \\
\partial_{t} g^{\prime}(x, t)=-2 \operatorname{Ric}^{\prime}(x, t)
\end{array}\right.\right.
$$

Throughout this paper, we denote by $\|\cdot\|$ the norm on $M^{\prime}$ with respect to $g^{\prime}$. Suppose that $u=u(x, t)$ solves the conjugate heat equation and satisfies $0<u<A$ in the geodesic cube $\mathcal{Q}_{2 \rho} \subset M^{\prime}$ as defined by

$$
\mathcal{Q}_{2 \rho, T}:=\left\{(x, t) \in M^{\prime} \times(0, T]: d(x, y, t) \leq 2 \rho\right\} .
$$

We set $\omega_{i}:=\partial_{i} \omega, \omega_{i j}:=\partial_{i} \partial_{j} \omega$, and so on, for some smooth function $\omega$ on $M^{\prime}$, where $1 \leq i, j \leq n$. Then we have:

Theorem 4.1. Let $(M, g)$ be a screen integrable globally null manifold and let $\left(M^{\prime}, g^{\prime}\right)$ be a leaf of $S(T M)$. Suppose that $\left(M^{\prime}, g^{\prime}(t)\right)$ (with $\left.t \in(0, T]\right)$ is a complete solution to the backward degenerate Ricci flow with $S c a l^{\prime} \geq-\rho_{1}$, $\operatorname{Ric}^{\prime} \geq-\rho_{2}$, and $\left\|\bar{\nabla} S c a l^{\prime}\right\| \leq \rho_{3}$, for some constants $\rho_{1}, \rho_{2}, \rho_{3} \geq 0$. Let $u=u(x, t)$ be any positive solution to the heat equation defined in $\mathcal{Q}_{2 \rho, T} \subset\left(M^{\prime} \times(0, T]\right)$ satisfying $0<u \leq A$. 
Then, there exist absolute constants $c_{1}, c_{2}$ depending on $n$ such that

$$
\frac{\|\bar{\nabla} u\|^{2}}{u^{2}} \leq\left(1+\ln \left(\frac{A}{u}\right)\right)^{2}\left(\frac{1}{t}+c_{2} \rho_{1}+4 \rho_{2}+2 \rho_{3}+\frac{1}{\rho^{2}}\left(\rho c_{1} \sqrt{\rho_{2}}+c_{2}\right)\right) .
$$

Proof. Let $f=\ln \frac{u}{A}$. It is easy to see that $1-f \geq 1$. Let $\phi=\|\bar{\nabla} \ln (1-f)\|^{2}=$ $\frac{\|\bar{\nabla} f\|^{2}}{(1-f)^{2}}$. Then, a straightforward calculation using 4.1) gives the evolution equation of $f$ as $\partial_{t} f=\Delta f+\|\bar{\nabla} f\|^{2}-S c a l^{\prime}$. Next, we compute the evolution equation of $\phi$. To that end, differentiating $\phi$ with respect to $t$ and using the evolution equation of $f$, gives

$$
\partial_{t} \phi=\frac{\partial}{\partial t}\left(\frac{\|\bar{\nabla} f\|^{2}}{(1-f)^{2}}\right)=\frac{\partial_{t}\|\bar{\nabla} f\|^{2}}{(1-f)^{2}}+\frac{2\|\bar{\nabla} f\|^{2} \partial_{t} f}{(1-f)^{3}} .
$$

On the other hand, the term $\partial_{t}\left(\|\bar{\nabla} f\|^{2}\right)$ is given by

$$
\begin{aligned}
\partial_{t}\left(\|\bar{\nabla} f\|^{2}\right)= & \left(\partial_{t} g^{i j}\right) \partial_{i} f \partial_{j} f+2 g^{\prime}\left(\bar{\nabla} f, \bar{\nabla} \partial_{t} f\right) \\
= & -2 \operatorname{Ric}_{i j}^{\prime} \partial_{i} f \partial_{j} f+2 g^{\prime}\left(\bar{\nabla} f, \bar{\nabla}\left(\underline{\Delta} f+\|\bar{\nabla} f\|^{2}-S c a l^{\prime}\right)\right) \\
= & -2 \operatorname{Ric}_{i j}^{\prime} \bar{\nabla}_{i} f \bar{\nabla}_{j} f+2 g^{\prime}(\bar{\nabla} f, \bar{\nabla} \underline{\Delta} f)+2 g^{\prime}\left(\bar{\nabla} f, \bar{\nabla}\|\bar{\nabla} f\|^{2}\right) \\
& -2 g^{\prime}\left(\bar{\nabla} f, \bar{\nabla} S c a l^{\prime}\right),
\end{aligned}
$$

in which we have used (4.1) and the evolution equation of $f$. Applying (4.3) to (4.2) and using the evolution equation of $f$, gives

$$
\begin{aligned}
\partial_{t} \phi= & \frac{-2 \operatorname{Ric}_{i j}^{\prime} \bar{\nabla}_{i} f \bar{\nabla}_{j} f+2 g^{\prime}(\bar{\nabla} f, \bar{\nabla} \underline{\Delta} f)+2 g^{\prime}\left(\bar{\nabla} f, \bar{\nabla}\|\bar{\nabla} f\|^{2}\right)-2 g^{\prime}\left(\bar{\nabla} f, \bar{\nabla} S c a l^{\prime}\right)}{(1-f)^{2}} \\
& +\frac{2\|\bar{\nabla} f\|^{2}\left(\underline{\underline{ }} f+\|\bar{\nabla} f\|^{2}-S c a l^{\prime}\right)}{(1-f)^{3}} \\
= & -\frac{2 \operatorname{Ric}_{i j}^{\prime} \bar{\nabla}_{i} f \bar{\nabla}_{j} f}{(1-f)^{2}}+\frac{2 f_{j} f_{j j i}}{(1-f)^{2}}+\frac{2 g^{\prime}\left(\bar{\nabla} f, \bar{\nabla}\|\bar{\nabla} f\|^{2}\right)}{(1-f)^{2}}-\frac{2 g^{\prime}\left(\bar{\nabla} f, \bar{\nabla} S c a l^{\prime}\right)}{(1-f)^{2}} \\
& +\frac{2\|\bar{\nabla} f\|^{2} \underline{\Delta} f}{(1-f)^{3}}+\frac{2\|\bar{\nabla} f\|^{4}}{(1-f)^{3}}-\frac{2 S c a l^{\prime}\|\bar{\nabla} f\|^{2}}{(1-f)^{3}} .
\end{aligned}
$$

In view of the Bochner-Weitzenböck identity (see [11] for details), we have that $\underline{\Delta}\left(\|\bar{\nabla} f\|^{2}\right)=-2 f_{i j}^{2}+2 g^{\prime}(\bar{\nabla} f, \bar{\nabla} \underline{\Delta} f)+2 \operatorname{Ric}_{i j}^{\prime} f_{i} f_{j}$, so that 4.3 reduces to

$$
\begin{aligned}
& \left(\partial_{t}-\underline{\Delta}\right)\|\bar{\nabla} f\|^{2} \\
& \quad=-2 f_{i j}^{2}+2 g^{\prime}\left(\bar{\nabla} f, \bar{\nabla}\|\bar{\nabla} f\|^{2}\right)-2 g^{\prime}\left(\bar{\nabla} f, \bar{\nabla} S c a l^{\prime}\right)-4 \operatorname{Ric}_{i j}^{\prime} \bar{\nabla}_{i} f \bar{\nabla}_{j} f .
\end{aligned}
$$

Next, we compute $\underline{\Delta} \phi$. To that end, a direct calculation gives

$$
\bar{\nabla} \phi=\frac{2 g^{\prime}(\bar{\nabla} f, \bar{\nabla} \bar{\nabla} f)}{(1-f)^{2}}+\frac{2\|\bar{\nabla} f\|^{2} \bar{\nabla} f}{(1-f)^{3}}=\frac{2 f_{i} f_{i j}}{(1-f)^{2}}+\frac{2 f_{i}^{2} f_{j}}{(1-f)^{3}} .
$$


Applying the definition of $\underline{\Delta}$ and relation 4.5 , we get

$$
\begin{aligned}
\Delta \phi & =\left(\frac{2 f_{i} f_{i j}}{(1-f)^{2}}\right)_{j}+\left(\frac{2 f_{i}^{2} f_{j}}{(1-f)^{3}}\right)_{j} \\
& =\frac{2 f_{i j} f_{i j}+f_{i} f_{i j j}}{(1-f)^{2}}+\frac{4(1-f) f_{i} f_{i j} f_{j}}{(1-f)^{4}}+\frac{4 f_{i} f_{i j} f_{j}+2 f_{i}^{2} f_{j j}}{(1-f)^{3}}+\frac{6 f_{i}^{2} f_{j}^{2}(1-f)}{(1-f)^{6}} \\
& =\frac{2 f_{i j}^{2}}{(1-f)^{2}}+\frac{2 f_{i} f_{i j j}}{(1-f)^{2}}+\frac{4 f_{i} f_{i j} f_{j}}{(1-f)^{3}}+\frac{4 f_{i} f_{i j} f_{j}}{(1-f)^{3}}+\frac{2 f_{i}^{2} f_{i j}}{(1-f)^{3}}+\frac{6 f_{i}^{2} f_{j}^{2}}{(1-f)^{4}},
\end{aligned}
$$

from which we deduce that

$$
\begin{aligned}
\left(\partial_{t}-\underline{\Delta}\right) \phi= & \frac{2 \operatorname{Ric}_{i j}^{\prime} f_{i} f_{j}}{(1-f)^{2}}+\frac{2 f_{j} f_{j j i}}{(1-f)^{2}}+\frac{2 g^{\prime}\left(\bar{\nabla} f, \bar{\nabla}\|\bar{\nabla} f\|^{2}\right)}{(1-f)^{2}}-\frac{2 g^{\prime}\left(\bar{\nabla} f, \bar{\nabla} S c a l^{\prime}\right)}{(1-f)^{2}} \\
& +\frac{2\|\bar{\nabla} f\|^{4}}{(1-f)^{3}}-\frac{2 S c a l^{\prime}\|\bar{\nabla} f\|^{2}}{(1-f)^{3}}-\frac{2 f_{i j}^{2}}{(1-f)^{2}}-\frac{2 f_{i} f_{i j j}}{(1-f)^{2}}-\frac{4 f_{i} f_{i j} f_{j}}{(1-f)^{3}} \\
& -\frac{4 f_{i} f_{i j} f_{j}}{(1-f)^{2}}-\frac{6 f_{i}^{2} f_{j}^{2}}{(1-f)^{4}}
\end{aligned}
$$

Rearranging the terms in 4.6 gives

$$
\begin{aligned}
\left(\partial_{t}-\underline{\Delta}\right) \phi= & -\frac{2}{(1-f)^{2}}\left\{f_{i j}^{2}+\frac{2 f_{i} f_{i j} f_{j}}{1-f}+\frac{f_{i}^{2} f_{j}^{2}}{(1-f)^{2}}\right\} \\
& -\left\{\frac{2 \operatorname{Ric}_{i j}^{\prime} f_{i} f_{j}}{(1-f)^{2}}+\frac{2 f_{j} f_{j j i}}{(1-f)^{2}}-\frac{2 f_{i} f_{i j j}}{(1-f)^{2}}\right\} \\
& -\left\{\frac{4 f_{i} f_{i j} f_{j}}{(1-f)^{3}}-\frac{4 f_{i}^{2} f_{j}^{2}}{(1-f)^{4}}+\frac{2 g^{\prime}\left(\bar{\nabla} f, \bar{\nabla}\|\bar{\nabla} f\|^{2}\right)}{(1-f)^{2}}\right\} \\
& -\left\{\frac{2 g^{\prime}\left(\bar{\nabla} f, \bar{\nabla} S c a l^{\prime}\right)}{(1-f)^{2}}-\frac{2 S c a l^{\prime}\|\bar{\nabla} f\|^{2}}{(1-f)^{3}}+\frac{2\|\bar{\nabla} f\|^{4}}{(1-f)^{3}}\right\} .
\end{aligned}
$$

We can simplify some terms in the braces of 4.7 further. The first term becomes

$$
-\frac{2}{(1-f)^{2}}\left\{f_{i j}^{2}+\frac{2 f_{i} f_{i j} f_{j}}{(1-f)}+\frac{f_{i}^{2} f_{j}^{2}}{(1-f)^{2}}\right\}=-\frac{2}{(1-f)^{2}}\left(f_{i j}+\frac{f_{i} f_{j}}{1-f}\right)^{2} .
$$

Using the Ricci identity on the next three terms we have

$$
-\frac{2 \operatorname{Ric}_{i j}^{\prime} f_{i} f_{j}}{(1-f)^{2}}+\frac{2 f_{j} f_{j j i}}{(1-f)^{2}}-\frac{2 f_{i} f_{i j j}}{(1-f)^{2}}=-\frac{4 \operatorname{Ric}_{i j}^{\prime} f_{i} f_{j}}{(1-f)^{2}},
$$

since the Ricci identity implies that $f_{j} f_{j j i}-f_{i} f_{i j j}=f_{j}\left(f_{j j i}-f_{i j j}\right)=-\operatorname{Ric}_{i j}^{\prime} f_{i} f_{j}$. Also

$$
-\frac{4 f_{i} f_{i j} f_{j}}{(1-f)^{3}}-\frac{4 f_{i}^{2} f_{j}^{2}}{(1-f)^{4}}=\frac{-2}{1-f} f_{j}\left(\frac{2 f_{i} f_{i j}}{(1-f)^{2}}+\frac{2 f_{i}^{2} f_{j}}{(1-f)^{3}}\right)=\frac{-2}{1-f} g^{\prime}(\bar{\nabla} f, \bar{\nabla} \phi) .
$$


Similarly, we have $2(1-f)^{-2} g^{\prime}\left(\bar{\nabla} f, \bar{\nabla}\|\bar{\nabla} f\|^{2}\right)=2 g^{\prime}(\bar{\nabla} f, \bar{\nabla} \phi)$, and then the second to the last three terms gives

$$
\begin{aligned}
-\frac{4 f_{i} f_{i j} f_{j}}{(1-f)^{3}}-\frac{4 f_{i}^{2} f_{j}^{2}}{(1-f)^{4}} & +\frac{2 g^{\prime}\left(\bar{\nabla} f, \bar{\nabla}\|\bar{\nabla} f\|^{2}\right)}{(1-f)^{2}} \\
& =\frac{-2}{1-f} g^{\prime}(\bar{\nabla} f, \bar{\nabla} \phi)+2 g^{\prime}(\bar{\nabla} f, \bar{\nabla} \phi)=\frac{-2}{1-f} g^{\prime}(\bar{\nabla} f, \bar{\nabla} \phi) .
\end{aligned}
$$

Putting all these together in (4.7), we get

$$
\begin{aligned}
\left(\partial_{t}-\underline{\Delta}\right) \phi= & -\frac{2}{(1-f)^{2}}\left(f_{i j}+\frac{f_{i} f_{j}}{1-f}\right)^{2}-\frac{2 f}{1-f} g^{\prime}(\bar{\nabla} f, \bar{\nabla} \phi) \\
& -\frac{4 \operatorname{Ric}_{i j}^{\prime} f_{i} f_{j}}{(1-f)^{2}}-\frac{2 g^{\prime}\left(\bar{\nabla} f, \bar{\nabla} S c a l^{\prime}\right)}{(1-f)^{2}}-\frac{2 S c a l^{\prime}\|\bar{\nabla} f\|^{2}}{(1-f)^{3}}-\frac{2\|\bar{\nabla} f\|^{4}}{(1-f)^{3}} .
\end{aligned}
$$

Then, using the curvature conditions we are left with the inequality

$$
\begin{aligned}
\left(\partial_{t}-\underline{\Delta}\right) \phi \leq & -\frac{2 f}{1-f} g^{\prime}(\bar{\nabla} f, \bar{\nabla} \phi)+\frac{2}{(1-f)} \rho_{1} \phi+4 \rho_{2} \phi \\
& +2 \rho_{3} \phi^{\frac{1}{2}}-2(1-f) \phi^{2} .
\end{aligned}
$$

Next, we apply a cut-off function in order to derive the desired estimate. To that end, let $\psi$ be a smooth cut function defined on $[0, \infty)$ such that $0 \leq \psi(s) \leq 1$, with $\psi^{\prime}(s) \leq 0, \psi^{\prime \prime}(s) \geq c_{1}$, and $\left|\psi^{\prime}\right|^{2} / \psi \leq-c_{2}$, for some constants $c_{1}, c_{2}>0$ depending on the dimension of the manifold only. Let us define a distance function $d(p, x)$ between the point $p$ and $x$ such that $\varphi(x, t)=\varphi(d(p, x, t))=\psi\left(d_{g^{\prime}(t)}(p, x) / \rho\right)$, for a smooth function $\varphi: M^{\prime} \times(0, T] \rightarrow \mathbb{R}$. It is easily seen that $\varphi(x, t)$ has its support in the closure of $\mathcal{Q}_{2 \rho, T}$. We note that $\varphi(x, t)$ is smooth at $(y, s) \in M^{\prime} \times(0, T]$, whenever point $y$ does not either coincide with $p$ or fall in the cut locus of $p$, with respect to the metric $g^{\prime}(y, s)$. In what follows, we consider the function $\varphi \phi$ defined in $\mathcal{Q}_{2 \rho, T} \times[0, \infty)$ is $C^{2}$ at the maxima. This assumption is supported by a standard argument by Calabi known as Calabi's trick (see [4 for more details). This approach is used in [5]; see also [11, 23]. Therefore, we obtain

$$
\frac{\|\bar{\nabla} \varphi\|^{2}}{\varphi}=\frac{\left\|\psi^{\prime}\right\|^{2}\|\bar{\nabla} d\|^{2}}{\rho^{2} \varphi} \leq \frac{c_{2}}{\rho^{2}}, \quad \frac{\partial \varphi}{\partial t} \leq c_{2} \rho_{1},
$$

and by the Laplacian comparison theorem (see [1] for more details), we have

$$
\underline{\Delta} \varphi=\frac{\psi^{\prime} \underline{\Delta} d}{\rho}+\frac{\psi^{\prime \prime}\|\bar{\nabla} d\|^{2}}{\rho} \geq \frac{c_{1}}{\rho}(n-1) \sqrt{\rho_{2}} \operatorname{coth}\left(\sqrt{\rho_{2}} \rho\right)-\frac{c_{2}}{\rho^{2}},
$$

which implies that $-\underline{\varphi} \leq \frac{1}{\rho^{2}}\left(c_{1} \sqrt{\rho_{2}} \rho \operatorname{coth}\left(\sqrt{\rho_{2}} \rho\right)+c_{2}\right)$.

Let $\left(x_{0}, t_{0}\right)$ be a point in $\mathcal{Q}_{2 \rho, T}$ at which $F=\varphi \phi$ attains its maximum value. At this point we have to assume that $F$ is positive. Note that if $F=0$ then $\varphi \phi\left(x_{0}, t_{0}\right)=0$ and hence, $\phi(x, t)=0$, for all $x \in M$ such that $d\left(x, x_{0}, t\right)<2 \rho$. This yields $\bar{\nabla} u(x, t)=0$ and the theorem will follow trivially at $(x, t)$. The approach 
here is to estimate $\left(\partial_{t}-\underline{\Delta}\right)(t F)$ and do some analyses on the result at the maximum point. To that end, we have

$$
\begin{aligned}
\left(\partial_{t}-\underline{\Delta}\right)(t F) & =F+t\left(\partial_{t}-\underline{\Delta}\right)(\varphi \phi) \\
& =F-2 t g^{\prime}(\bar{\nabla} \varphi, \bar{\nabla} \phi)+t \varphi\left(\partial_{t}-\underline{\Delta}\right) \phi+t \phi\left(\partial_{t}-\underline{\Delta}\right) \varphi .
\end{aligned}
$$

Note that at the maximum point $\left(x_{0}, t_{0}\right)$, we have by the derivative test that

$$
\underline{\Delta} F\left(x_{0}, t_{0}\right)=0, \quad \partial_{t} F\left(x_{0}, t_{0}\right) \geq 0, \quad \text { and } \quad \underline{\Delta} F\left(x_{0}, t_{0}\right) \leq 0 .
$$

Taking $t F$ on $M^{\prime} \times(0, T]$, we have $\left(\partial_{t}-\underline{\Delta}\right)(t F) \geq 0$, whenever $(t F)$ achieves its maximum. Similarly, by this argument, we have $\bar{\nabla}(\varphi \phi)\left(x_{0}, t_{0}\right)-\phi \bar{\nabla} \varphi\left(x_{0}, t_{0}\right)=$ $\varphi \bar{\nabla} \phi\left(x_{0}, t_{0}\right)$, which means $\varphi \bar{\nabla} \phi$ can always be replaced by $-\phi \bar{\nabla} \varphi$. By 4.8, 4.11, and 4.12, one has

$$
\begin{aligned}
F-2 t g^{\prime}(\bar{\nabla} \varphi, \bar{\nabla} \phi)+t \varphi-2 f & (1-f)^{-1} g^{\prime}(\bar{\nabla} f, \bar{\nabla} \phi)+2(1-f)^{-1} \rho_{1} \phi \\
& +4 \rho_{2} \phi+2 \rho_{3} \phi^{\frac{1}{2}}+2(1-f) \phi^{2}+t \phi\left(\partial_{t}-\underline{\Delta}\right) \varphi \geq 0
\end{aligned}
$$

Taking $0 \leq \varphi \leq 1$ and noticing that $\frac{1}{1-f} \leq 1$, the last inequality becomes

$$
\begin{aligned}
F & -2 t g^{\prime}\left(\frac{\bar{\nabla} \varphi}{\varphi}, \bar{\nabla}(\varphi \phi)\right)-2 t \frac{\|\bar{\nabla} \varphi\|^{2}}{\varphi} \phi \\
& +t\left\{2 \rho_{1} F+4 \rho_{2} F+2 \rho_{3} F^{\frac{1}{2}}+2(1-f) F^{2}\right\} \\
& -2 t f(1-f)^{-1}\|\bar{\nabla} f\|\|\bar{\nabla} \varphi\| \phi+t \phi\left(\partial_{t}-\underline{\Delta}\right) \varphi \geq 0 .
\end{aligned}
$$

Applying the relation $2 \rho_{3} F^{\frac{1}{2}} \leq \rho_{3} F+\rho_{3}$ (see [11] for details) and using Young's inequality, we derive

$$
\begin{aligned}
-2 t f(1-f)^{-1}\|\bar{\nabla} f\|\|\bar{\nabla} \varphi\| \phi & \leq t \phi\left(\frac{\|\bar{\nabla} f\|^{2}}{1-f} \varphi+\frac{\|\bar{\nabla} \varphi\|^{2}}{\varphi} \frac{f^{2}}{1-f}\right) \\
& =t(1-f) F^{2}+t F \frac{c_{2}}{\rho^{2}} \frac{f^{2}}{1-f} .
\end{aligned}
$$

Notice also that by the bounds given in 4.9 and 4.10 , we have

$$
\begin{aligned}
t \phi\left(\partial_{t}-\underline{\Delta}\right) \varphi & \leq t \phi\left(c_{2} \rho_{1}+\frac{1}{\rho^{2}}\left(c_{1} \sqrt{\rho_{2}} \operatorname{coth}\left(\sqrt{\rho_{2} \rho}\right)+c_{2}\right)\right) \\
& \leq t \phi\left(c_{2} \rho_{1}+\frac{1}{\rho^{2}}\left(\rho c_{1} \sqrt{\rho_{2}}+c_{2}\right)\right)
\end{aligned}
$$

Putting these together and dividing through by $(1-f)$, while noticing that $\frac{1}{1-f} \leq 1$ and $\frac{-f}{1-f} \leq 1$, we have

$$
\begin{aligned}
F-2 t \frac{c_{2}}{\rho^{2}} F+t\left(2 \rho_{1}+4 \rho_{2}\right. & \left.+\rho_{3}\right) F+t \rho_{3} \\
& +t F \frac{c_{2}}{\rho^{2}}-t F^{2}+t \phi\left(c_{2} \rho_{1}+\frac{1}{\rho^{2}}\left(\rho c_{1} \sqrt{\rho_{2}}+c_{2}\right)\right) \geq 0 .
\end{aligned}
$$


Therefore, we have

$$
\begin{aligned}
t F^{2} \leq & F+t\left(2 \rho_{1}+4 \rho_{2}+\rho_{3}\right) F+t\left(c_{2} \rho_{1}+\frac{1}{\rho^{2}}\left(\rho c_{1} \sqrt{\rho_{2}}+c_{2}\right)\right) F \\
& -t \frac{c_{2}}{\rho^{2}} F+t \rho_{3} .
\end{aligned}
$$

Then,

$$
F^{2} \leq F\left\{\frac{1}{t}+c_{2} \rho_{1}+4 \rho_{2}+\rho_{3}+\frac{1}{\rho^{2}}\left(\rho c_{1} \sqrt{\rho_{2}}+c_{2}\right)+\rho_{3}\right\}+\rho_{3} .
$$

From here we can conclude that

$$
F \leq \frac{1}{t}+c_{2} \rho_{1}+4 \rho_{2}+\rho_{3}+\sqrt{\rho_{3}}+\frac{1}{\rho^{2}}\left(\rho c_{1} \sqrt{\rho_{2}}+c_{2}\right)
$$

at $\left(x_{0}, t_{0}\right)$. Therefore,

$$
\|\bar{\nabla} f\|^{2} \leq(1-f)^{2}\left(\frac{1}{t}+c_{2} \rho_{1}+4 \rho_{2}+2 \rho_{3}+\frac{1}{\rho^{2}}\left(\rho c_{1} \sqrt{\rho_{2}}+c_{2}\right)\right),
$$

which completes the proof.

The boundedness assumption may be weakened in the case of the forward degenerate Ricci-type flow. Therefore, we have the following.

Theorem 4.2. Let $(M, g)$ be a screen integrable globally null manifold and let $\left(M^{\prime}, g^{\prime}\right)$ be a leaf of $S(T M)$. Suppose that $\left(M^{\prime}, g^{\prime}(t)\right)$ (with $\left.t \in(0, T]\right)$ is a complete solution to the forward degenerate Ricci-type flow with $S c a l^{\prime} \geq-\rho_{1}$ and $\left\|\bar{\nabla} S c a l^{\prime}\right\| \leq$ $\rho_{3}$, for some constants $\rho_{1}, \rho_{3} \geq 0$. Let $u=u(x, t)$ be any positive solution to the heat equation defined in $\mathcal{Q}_{2 \rho, T} \subset\left(M^{\prime} \times(0, T]\right)$ satisfying $0<u \leq A$. Then there exist absolute constants $c_{1}, c_{2}$ depending on $n$ such that

$$
\frac{\|\bar{\nabla} u\|^{2}}{u^{2}} \leq\left(1+\ln \left(\frac{A}{u}\right)\right)^{2}\left(\frac{1}{t}+c_{2} \rho_{1}+2 \rho_{3}+\frac{c_{2}}{\rho^{2}}\right) .
$$

Proof. The proof of this theorem is similar to that of Theorem 4.1. The disparity between the estimate inequalities in Theorem 4.1 and Theorem 4.2 arises through their respective proofs, which we show as follows. Similary, set $f=\ln \frac{u}{A}$ and $\phi=\|\bar{\nabla} \ln (1-f)\|^{2}=\frac{\|\bar{\nabla} f\|^{2}}{(1-f)^{2}}$. Notice that $g^{\prime}(t)$ evolves by the forward degenerate Ricci flow, where the inverse metric evolves as $\partial_{t}\left(g^{\prime i j}\right)=2 \operatorname{Ric}_{i j}^{\prime}$ and then

$$
\begin{aligned}
\partial_{t}\left(\|\bar{\nabla} f\|^{2}\right)= & 2 \operatorname{Ric}_{i j}^{\prime} \bar{\nabla}_{i} f \bar{\nabla}_{j} f+2 g^{\prime}(\bar{\nabla} f, \bar{\nabla} \underline{\Delta f})+2 g^{\prime}\left(\bar{\nabla} f, \bar{\nabla}\|\bar{\nabla} f\|^{2}\right) \\
& -2 g^{\prime}\left(\bar{\nabla} f, \bar{\nabla} S c a l^{\prime}\right) ;
\end{aligned}
$$


hence, the counterpart of 4.7 is

$$
\begin{aligned}
\left(\partial_{t}-\underline{\Delta}\right) \phi= & -\frac{2}{(1-f)^{2}}\left\{f_{i j}^{2}+\frac{2 f_{i} f_{i j} f_{j}}{1-f}+\frac{f_{i}^{2} f_{j}^{2}}{(1-f)^{2}}\right\} \\
& +\left\{\frac{2 \operatorname{Ric}_{i j}^{\prime} f_{i} f_{j}}{(1-f)^{2}}+\frac{2 f_{j} f_{j j i}}{(1-f)^{2}}-\frac{2 f_{i} f_{i j j}}{(1-f)^{2}}\right\} \\
& -\left\{\frac{4 f_{i} f_{i j} f_{j}}{(1-f)^{3}}-\frac{4 f_{i}^{2} f_{j}^{2}}{(1-f)^{4}}+\frac{2 g^{\prime}\left(\bar{\nabla} f, \bar{\nabla}\|\bar{\nabla} f\|^{2}\right)}{(1-f)^{2}}\right\} \\
& -\left\{\frac{2 g^{\prime}\left(\bar{\nabla} f, \bar{\nabla} S c a l^{\prime}\right)}{(1-f)^{2}}-\frac{2 S c a l^{\prime}\|\bar{\nabla} f\|^{2}}{(1-f)^{3}}+\frac{2\|\bar{\nabla} f\|^{4}}{(1-f)^{3}}\right\}
\end{aligned}
$$

Now, using the Ricci identity, some braced terms vanish. Then we can conclude that

$$
\begin{aligned}
\left(\partial_{t}-\underline{\Delta}\right) \phi= & -\frac{2}{(1-f)^{2}}\left(f_{i j}+\frac{f_{i} f_{j}}{1-f}\right)^{2}-\frac{2 f}{1-f} g^{\prime}(\bar{\nabla} f, \bar{\nabla} \phi) \\
& +\frac{4 \operatorname{Ric}_{i j}^{\prime} f_{i} f_{j}}{(1-f)^{2}}-\frac{2 g^{\prime}\left(\bar{\nabla} f, \bar{\nabla} S c a l^{\prime}\right)}{(1-f)^{2}}-\frac{2 S c a l^{\prime}\|\bar{\nabla} f\|^{2}}{(1-f)^{3}}-\frac{2\|\bar{\nabla} f\|^{4}}{(1-f)^{3}} .
\end{aligned}
$$

Using the curvature conditions, we are left with the following inequality as in 4.8 ):

$$
\left(\partial_{t}-\underline{\Delta}\right) \phi \leq-\frac{2 f}{1-f} g^{\prime}(\bar{\nabla} f, \bar{\nabla} \phi)+\frac{2}{(1-f)} \rho_{1} \phi+2 \rho_{3} \phi^{\frac{1}{2}}-2(1-f) \phi^{2} .
$$

The rest of the proof follows as in that of Theorem 4.1 .

\section{Gradient estimates on forward heat equation}

Let $(M, g)$ be a screen integrable globally null manifold and let $\left(M^{\prime}, g^{\prime}\right)$ be an $n$-dimensional complete Riemannian integral manifold of the screen distribution $S(T M)$ without boundary. Next, we discuss space-time gradient estimates for positive solutions of the forward heat equation along the degenerate Ricci flow. More precisely, we consider

$$
\partial_{t} g^{\prime}(x, t)=-2 \operatorname{Ric}^{\prime}(x, t), \quad(x, t) \in M^{\prime} \times(0, T],
$$

coupled to

$$
\left(\underline{\Delta}-\partial_{t}\right) u(x, t)=0, \quad(x, t) \in M^{\prime} \times(0, T] .
$$

As mentioned in [1, in general, our degenerate version of the estimates is also a local one and obtained in a geodesic cube. We will show how this local estimate can lead to achieving a global one. We start by proving the following lemma, which is useful for this section. Let us define the geodesic cube

$$
\mathcal{Q}_{2 \rho, T}:=\left\{(x, t) \in M^{\prime} \times(0, T]: d\left(x, x_{0}, t\right) \leq \rho\right\} .
$$

Lemma 5.1. Let $(M, g)$ be a screen integrable globally null manifold and let $\left(M^{\prime}, g^{\prime}\right)$ be a leaf of $S(T M)$. Suppose that $\left(M^{\prime}, g^{\prime}(t)\right)$ is a complete solution to the forward degenerate Ricci flow in some time interval $(0, T]$, and $-\rho_{1} g^{\prime} \leq \operatorname{Ric}^{\prime} \leq$ 
$\rho_{2} g^{\prime}$, for some positive constants $\rho_{1}$ and $\rho_{2}$. For any smooth positive solution $u \in C^{2,1}\left(M^{\prime} \times(0, T]\right)$ to the heat equation in the geodesic cube $\mathcal{Q}_{2 \rho, T}$, it holds that

$$
\begin{aligned}
\left(\underline{\Delta}-\partial_{t}\right) G \geq & -2 g^{\prime}(\bar{\nabla} f, \bar{\nabla} G)\left(\|\bar{\nabla} f\|^{2}-\alpha \partial_{t} f\right)-2 \alpha t \rho_{1}\|\bar{\nabla} f\|^{2} \\
& +\frac{2 \alpha}{n p} t\left(\|\bar{\nabla} f\|^{2}-\partial_{t} f\right)^{2}-\frac{\alpha n q}{2} t\left(\rho_{1}+\rho_{2}\right)^{2}
\end{aligned}
$$

where $f=\ln u, G=t\left(\|\bar{\nabla} f\|^{2}-\alpha \partial_{t} f\right)$, and $\alpha \geq 1$ are given such that $\frac{1}{p}+\frac{1}{q}=\frac{1}{\alpha}$, for any real numbers $p, q>0$.

Proof. We have $\underline{\Delta} G=t\left(\underline{\Delta}\|\bar{\nabla} f\|^{2}-\alpha \underline{\Delta} \partial_{t} f\right)$ and $\partial_{t} G=\left(\|\bar{\nabla} f\|^{2}-\alpha \partial_{t} f\right)+$ $t \partial_{t}\left(\|\bar{\nabla} f\|^{2}-\alpha \partial_{t} f\right)$. In local coordinates and using the Einstein summation convention, we have, by Bochner-Weitzenböck's identity, $\Delta\|\bar{\nabla} f\|^{2}=2 f_{i j}^{2}+2 f_{j} f_{j j i}+$ $2 \operatorname{Ric}_{i j}^{\prime} f_{i} f_{j}$. By the hypothesis of the lemma that $g^{\prime}(x, t)$ evolves by the degenerate Ricci flow, we have $\partial_{t}\left(\|\bar{\nabla} f\|^{2}\right)=\left(\partial_{t} g^{\prime i j}\right) \partial_{i} f \partial_{j} f+2 g^{\prime i j} \partial_{i} f \partial_{j} \partial_{t} f=2 \operatorname{Ric}_{i j}^{\prime} f_{i} f_{j}+$ $2 f_{i} f_{t, i}$. Similarly, $\partial_{t}(\underline{\Delta} f)=\left(\partial_{t} g^{i j}\right) \partial_{i} \partial_{j} f+g^{i j} \partial_{i} \partial_{j} \partial_{t} f=2 \operatorname{Ric}_{i j}^{\prime} f_{i j}+\underline{\Delta}\left(\partial_{t} f\right)$, which implies that $\underline{\Delta}\left(\partial_{t} f\right)=\partial_{t}(\underline{\Delta} f)-2 \operatorname{Ric}_{i j}^{\prime} f_{i j}$. With the above computations, we obtain

$$
\begin{aligned}
\underline{\Delta} G & =t\left(2 f_{i j}^{2}+2 f_{i} f_{j j i}+2 \operatorname{Ric}_{i j}^{\prime} f_{i} f_{j}+2 \alpha \operatorname{Ric}_{i j}^{\prime} f_{i j}-\alpha \partial_{t}(\underline{\Delta} f)\right) \\
& \geq t\left[\left(2 f_{i j}^{2}+\alpha \operatorname{Ric}_{i j}^{\prime} f_{i j}\right)+2 f_{j} f_{j j i}-2 \rho_{1}\|\bar{\nabla} f\|^{2}-\alpha \partial_{t}(\underline{\Delta} f)\right]
\end{aligned}
$$

at an arbitrary point $(x, t) \in \mathcal{Q}_{2 \rho, T}$.

On the other hand, $\partial_{t} G=\left(\|\bar{\nabla} f\|^{2}-\alpha \partial_{t} f\right)+t\left(2 \operatorname{Ric}_{i j}^{\prime} f_{i} f_{j}+2 f_{i} f_{t, i}-\alpha \partial_{t} \partial_{t} f\right)$. Note that $f=\ln u$ implies the evolution $\partial_{t} f=\Delta f+\|\bar{\nabla} f\|^{2},(x, t) \in \mathcal{Q}_{2 \rho, T}$. Therefore, we have

$$
\begin{aligned}
& \left(\underline{\Delta}-\partial_{t}\right) G \geq t\left[\left(2 f_{i j}^{2}+2 \alpha \operatorname{Ric}_{i j}^{\prime} f_{i j}\right)+2 f_{j} f_{j j i}-2 \rho_{1}\|\bar{\nabla} f\|^{2}-\alpha \partial_{t}(\underline{\Delta} f)\right] \\
& -\left[\left(\|\bar{\nabla} f\|^{2}-\alpha \partial_{t} f\right)+t \partial_{t}\left(\|\bar{\nabla} f\|^{2}-\alpha \partial_{t} f\right)\right] \\
& =t\left(2 f_{i j}^{2}+2 \alpha \operatorname{Ric}_{i j}^{\prime} f_{i j}\right)-2 g^{\prime}(\bar{\nabla} f, \bar{\nabla} G) \\
& -\left(\|\bar{\nabla} f\|^{2}-\alpha \partial_{t} f\right)-2 \alpha t \rho_{1}\|\bar{\nabla} f\|^{2} \text {. }
\end{aligned}
$$

Now choosing any two real numbers $p, q>0$ such that $\frac{1}{p}+\frac{1}{q}=\frac{1}{\alpha}$, we can write

$$
2 f_{i j}^{2}+2 \alpha \operatorname{Ric}_{i j}^{\prime} f_{i j}=\frac{2 \alpha}{p} f_{i j}^{2}+2 \alpha\left(\frac{1}{q} f_{i j}^{2}+\operatorname{Ric}_{i j}^{\prime} f_{i j}\right) \geq \frac{2 \alpha}{p} f_{i j}^{2}-\frac{\alpha q}{2} \operatorname{Ric}_{i j}^{\prime 2},
$$

where we have used completing the square to arrive at the last inequality. Also by the Cauchy-Schwarz inequality, $(\underline{\Delta} f)^{2}=\left\|g^{\prime i j} \partial_{i} \partial_{j} f\right\|^{2} \leq n f_{i j}^{2}$ holds at an arbitrary point $(x, t) \in \mathcal{Q}_{2 \rho, T}$; therefore, we have $f_{i j}^{2} \geq \frac{1}{n}(\underline{\Delta} f)^{2}$. We can also write the boundedness condition on the degenerate Ricci curvature as $-\left(\rho_{1}+\rho_{2}\right) g^{\prime} \leq \operatorname{Ric}_{i j}^{\prime} \leq$ $\left(\rho_{1}+\rho_{2}\right) g^{\prime}$ so that $\sup _{M}\left\|\operatorname{Ric}_{i j}^{\prime}\right\|^{2} \leq n\left(\rho_{1}+\rho_{2}\right)^{2}$, since the Ricci curvature tensor is 
symmetric. Therefore, we have

$$
2 t\left(f_{i j}^{2}+2 \alpha \operatorname{Ric}_{i j}^{\prime} f_{i j}\right) \geq \frac{2 \alpha}{n p} t(\underline{\Delta} f)^{2}-\frac{\alpha n q}{2} t\left(\rho_{1}+\rho_{2}\right)^{2} .
$$

Hence the desired result. Our calculation is valid in the cube $\mathcal{Q}_{2 \rho, T}$.

Next, we state and prove a degenerate version result for local gradient estimates (space-time) for the positive solutions to the heat equation in the geodesic cube $\mathcal{Q}_{2 \rho, T}$ of bounded Ricci curvature manifolds evolving by the degenerate Ricci-type flow.

Theorem 5.2. Let $(M, g)$ be a screen integrable globally null manifold and let $\left(M^{\prime}, g^{\prime}\right)$ be a leaf of $S(T M)$. Suppose that $\left(M^{\prime}, g^{\prime}(t)\right)$, with $t \in(0, T]$, is a complete solution of the degenerate Ricci-type flow (5.1) such that the Ricci curvature is bounded in $\mathcal{Q}_{2 \rho, T}$, i.e., $-\rho_{1} g^{\prime}(x, t) \leq \operatorname{Ric}^{\prime}(x, t) \leq \rho_{2} g^{\prime}(x, t)$ for some positive constants $\rho_{1}$ and $\rho_{2}$, with $(x, t) \in \mathcal{Q}_{2 \rho, T} \subset\left(M^{\prime} \times(0, T]\right)$. If a smooth positive function $u \in C^{2,1}\left(M^{\prime} \times(0, T]\right)$ solves the heat equation $\sqrt{5.2}$ in the geodesic cube $\mathcal{Q}_{2 \rho, T}$, then for any given $\alpha>1$ with $\frac{1}{p}+\frac{1}{q}=\frac{1}{\alpha}$ and all $(x, t) \in \mathcal{Q}_{2 \rho, T} \times(0, T]$, the following estimate holds:

$$
\begin{aligned}
\sup _{x \in \mathcal{Q}_{2 \rho, T}}\left\{\|\bar{\nabla} f\|^{2}-\alpha \partial_{t} f\right\} \leq & \frac{\alpha n p}{4 t}+c \alpha^{2}\left(\frac{\alpha^{2} p}{\rho^{2}(\alpha-1)}+\frac{1}{t}+\left(\rho_{1}+\rho_{2}\right)\right) \\
& +\frac{\alpha^{2} n p}{2(\alpha-1)} \rho_{1}+\frac{\alpha n}{2}\left(\rho_{1}+\rho_{2}\right) \sqrt{p q},
\end{aligned}
$$

where $c$ is an arbitrary constant depending only on the dimension of the leaf.

Proof. Let $f=\ln u$ and $G=t\left(\|\bar{\nabla} f\|^{2}-\alpha \partial_{t} f\right)$. Then, $\frac{1}{t} G=\frac{\|\bar{\nabla} u\|^{2}}{u^{2}}-\alpha \frac{u_{t}}{u}$. We use the approach of applying a cut-off function and estimating $\left(\underline{\Delta}-\partial_{t}\right)(t \varphi G)$ at the point where the maximum value for $(\varphi G)$ is attained, as we did in Theorem 4.1 We have the following:

$$
\left(\underline{\Delta}-\partial_{t}\right)(t \varphi G)=2 t \bar{\nabla} \varphi \bar{\nabla} G-\varphi G+t \varphi\left(\underline{\Delta}-\partial_{t}\right) G+t G\left(\underline{\Delta}-\partial_{t}\right) \varphi
$$

Suppose that $\varphi G$ attains its maximum value at $\left(x_{0}, t_{0}\right) \in M^{\prime} \times(0, T]$ for $t_{0}>0$. Since $(\varphi G)(x, 0)=0$ for all $x \in M$, we have by the derivative test that

$$
\bar{\nabla}(\varphi G)\left(x_{0}, t_{0}\right)=0, \quad \frac{\partial}{\partial t}(\varphi G)\left(x_{0}, t_{0}\right) \geq 0, \quad \underline{\Delta}(\varphi G)\left(x_{0}, t_{0}\right) \leq 0,
$$

where the function $(\varphi G)$ is being considered with support on $\mathcal{Q}_{2 \rho, T} \times(0, T]$ and we have assumed that $(\varphi G)\left(x_{0}, t_{0}\right)>0$, for $t_{0}>0$. By (5.5) we notice that $\left(\underline{\Delta}-\partial_{t}\right)(\varphi G) \leq 0$. Using (5.4), 5.5), and Lemma 5.1, we have

$$
\begin{aligned}
0 \geq & \left(\underline{\Delta}-\partial_{t}\right)(t \varphi G) \geq 2 t \bar{\nabla} \varphi \bar{\nabla} G-\varphi G+t \varphi\left\{-2 g^{\prime}(\bar{\nabla} f, \bar{\nabla} G)-\left(\|\bar{\nabla} f\|^{2}-\alpha \partial_{t} f\right)\right. \\
& \left.-2 \alpha t \rho_{1}\|\bar{\nabla} f\|^{2}+\frac{2 \alpha}{n p} t\left(\|\bar{\nabla} f\|^{2}-\partial_{t} f\right)^{2}-\frac{\alpha n q}{2} t \widehat{\rho}\right\}+t G\left(\underline{\Delta}-\partial_{t}\right) \varphi,
\end{aligned}
$$


where $\widehat{\rho}=\rho_{1}+\rho_{2}$. Note also that $\varphi \bar{\nabla} G$ can be replaced by $-G \bar{\nabla} \varphi$, by the condition $\bar{\nabla}(\varphi G)=0$. Therefore, we have

$$
\begin{aligned}
0 \leq & 2 t \bar{\nabla} \varphi \bar{\nabla} G-\varphi G+2 t \bar{\nabla} f \bar{\nabla} \varphi G \\
& +t \varphi\left\{\frac{2 \alpha}{n p} t\left(\|\bar{\nabla} f\|^{2}-\partial_{t} f\right)^{2}-2 \alpha t \rho_{1}\|\bar{\nabla} f\|^{2}-\frac{\alpha n q}{2} t \widehat{\rho}\right\} \\
& -t \varphi\left(\|\bar{\nabla} f\|^{2}-\alpha \partial_{t} f\right)+t G\left(\underline{\Delta}-\partial_{t}\right) \varphi
\end{aligned}
$$

from which we get

$$
\begin{aligned}
0 \leq & -2 t \frac{\|\bar{\nabla} \varphi\|^{2}}{\varphi} G-2 \varphi G-2 t\|\bar{\nabla} f\|^{2}\|\bar{\nabla} \varphi\|^{2} G \\
& +t \varphi\left\{\frac{2 \alpha}{n p} t\left(\|\bar{\nabla} f\|^{2}-\partial_{t} f\right)^{2}-2 \alpha t \rho_{1}\|\bar{\nabla} f\|^{2}-\frac{\alpha n q}{2} t \widehat{\rho}\right\} \\
& +t G\left(\underline{\Delta}-\partial_{t}\right) \varphi .
\end{aligned}
$$

As we have noted earlier, Calabi's trick and the Laplacian comparison theorem allow us to do the following calculation on the cut-off function depending on the geodesic distance, since we know that the cut locus does not intersect with the geodesic cube $-\frac{\|\bar{\nabla} \varphi\|^{2}}{\varphi} \geq-\frac{c_{2}}{\rho^{2}}, \quad \underline{\varphi} \geq-\frac{c_{1}}{\rho} \sqrt{\rho_{1}}-\frac{c_{2}}{\rho^{2}}$, and $-\frac{\partial \varphi}{\partial t} \geq-c_{2} \widehat{\rho}-\frac{c_{1}}{\tau}$, $\tau \in(0, T]$. Hence, $t G\left(\underline{\Delta}-\partial_{t}\right) \varphi \geq t c_{3}\left(-\frac{1}{\rho} \sqrt{\rho_{1}}-\frac{1}{\rho^{2}}-\frac{1}{\tau}-\hat{\rho}\right)$, where we have taken $c_{3}$ to be the maximum of $c_{1}, c_{2}$, so our computation becomes

$$
\begin{aligned}
0 \geq & -2 t \frac{c_{2}}{\rho^{2}} G-2 \varphi G-2 t \frac{\sqrt{c_{2}}}{\rho}\|\bar{\nabla} f\| \varphi^{\frac{1}{2}} G \\
& +t \varphi\left\{\frac{2 \alpha}{n p} t\left(\|\bar{\nabla} f\|^{2}-\partial_{t} f\right)^{2}-2 \alpha t \rho_{1}\|\bar{\nabla} f\|^{2}-\frac{\alpha n q}{2} t \widehat{\rho}\right\} \\
& +t c_{3}\left(-\frac{1}{\rho} \sqrt{\rho_{1}}-\frac{1}{\rho^{2}}-\frac{1}{\tau}-\widehat{\rho}\right) G .
\end{aligned}
$$

Multiplying through by $\varphi$ such that $0 \leq \varphi \leq 1$, we have

$$
\begin{aligned}
0 \geq & -2 \varphi^{2} G-2 t \frac{\sqrt{c_{2}}}{\rho}\|\bar{\nabla} f\| \varphi^{\frac{3}{2}} G \\
& +\frac{2 t^{2}}{n}\left\{\frac{\alpha}{p}\left(\varphi\|\bar{\nabla} f\|^{2}-\varphi \partial_{t} f\right)^{2}-\alpha n \rho_{1} \varphi^{2}\|\bar{\nabla} f\|^{2}-\frac{\alpha n^{2} q}{4} t \widehat{\rho} \varphi^{2}\right\} \\
& +t c_{3}\left(-\frac{1}{\rho} \sqrt{\rho_{1}}-\frac{1}{\rho^{2}}-\frac{1}{\tau}-\widehat{\rho}\right)(\varphi G) .
\end{aligned}
$$

Using a standard argument from Lie and Yau [15] (see also Schoen and Yau [23] and references therein), we let $y=\varphi\|\bar{\nabla} f\|^{2}$ and $Z=\varphi \partial_{t} f$ to have $\varphi^{2}\|\bar{\nabla} f\|^{2}=\varphi y \leq y$, and $y^{\frac{1}{2}}(y-\alpha z)=\varphi^{\frac{1}{2}}\|\bar{\nabla} f\|\left(\varphi\|\bar{\nabla} f\|^{2}-\varphi \partial_{t} f\right)=\frac{1}{t}\|\bar{\nabla} f\| \varphi^{\frac{3}{2}} G$. Noting that

$$
(y-z)^{2}=\frac{1}{\alpha^{2}}(y-\alpha z)^{2}+\frac{\alpha-1}{\alpha^{2}} y^{2}+\frac{2(\alpha-1)}{\alpha^{2}} y(y-\alpha z)
$$


and

$$
\begin{aligned}
(y-z)^{2}-n p \rho_{1} y-n p \frac{\sqrt{c_{2}}}{\rho} y^{\frac{1}{2}}(y-\alpha z) & \\
= & \frac{1}{\alpha^{2}}(y-\alpha z)^{2}+\left(\frac{\alpha-1}{\alpha^{2}} y^{2}-n p \rho_{1} y\right) \\
& +\left(\frac{2(\alpha-1)}{\alpha^{2}} y-n p \frac{\sqrt{c_{2}}}{\rho} y^{\frac{1}{2}}\right)(y-\alpha z)
\end{aligned}
$$

and also using the inequality $a x^{2}-b x \geq-\frac{b^{2}}{4 a}(a, b>0)$, we have

$$
\begin{aligned}
& \frac{\alpha-1}{\alpha^{2}} y^{2}-n p \rho_{1} y \geq-\frac{\alpha^{2} n^{2} p^{2} \rho_{1}^{2}}{4(\alpha-1)^{2}} \text { and } \\
& \frac{2(\alpha-1)}{\alpha^{2}} y-n p \frac{\sqrt{c_{2}}}{\rho} y^{\frac{1}{2}} \geq-\frac{c_{2} \alpha^{2} n^{2} p^{2}}{8(\alpha-1) \rho^{2}} .
\end{aligned}
$$

Putting together (5.7)-5.10), the second and the third terms in the right hand side of (5.6) are further calculated as follows, using $t(y-\alpha z)=\varphi G$ :

$$
\begin{aligned}
& \frac{2 t^{2}}{n}\left\{\frac{\alpha}{p}(y-z)^{2}-\alpha n \rho_{1} y-\frac{\alpha n^{2} q}{4} \widehat{\rho} \varphi^{2}-\frac{n \sqrt{c_{2}}}{\rho} y^{\frac{1}{2}}(y-\alpha z)\right\} \\
& \quad \geq \frac{2 t^{2}}{n}\left\{\left(\frac{1}{\alpha p}(y-\alpha z)^{2}-\frac{\alpha^{3} n^{2} p \rho_{1}^{2}}{4(\alpha-1)^{2}}\right)-\left(\frac{c_{2} \alpha^{3} n^{2} p}{8 \rho^{2}(\alpha-1)}(y-\alpha z)+\frac{\alpha n^{2} q}{4} \widehat{\rho} \varphi^{2}\right)\right\} .
\end{aligned}
$$

Hence, by (5.6),

$$
\begin{array}{r}
\left\{\frac{2 t^{2}}{n}\left(\frac{1}{\alpha p}(y-\alpha z)^{2}-\frac{\alpha^{3} n^{2} p \rho_{1}^{2}}{4(\alpha-1)^{2}}\right)-\frac{2 t}{n}\left(\frac{c_{2} \alpha^{3} n^{2} p}{8 \rho^{2}(\alpha-1)}(\varphi G)\right)-\frac{\alpha n q}{2} t^{2} \widehat{\rho} \varphi^{2}\right\} \\
+t c_{3}\left(-\frac{1}{\rho} \sqrt{\rho_{1}}-\frac{1}{\rho^{2}}-\frac{1}{\tau}-\widehat{\rho}\right)(\varphi G)-2 \varphi^{2} G \leq 0 .
\end{array}
$$

Since $t(y-\alpha z)=\varphi G$, we have

$$
\begin{aligned}
0 \geq \frac{2}{\alpha n p}(\varphi G)^{2} & +\left[\frac{c_{4}}{\rho^{2}} t\left(-\frac{p \alpha^{3}}{(\alpha-1)}-\rho \sqrt{\rho_{1}}-1-\frac{\rho^{2}}{\tau}-\rho^{2} \widehat{\rho}\right)-1\right](\varphi G) \\
& -\left(\frac{\alpha^{3} n p \rho_{1}^{2}}{2(\alpha-1)^{2}} t^{2}+\frac{\alpha n q}{2} t^{2} \widehat{\rho} \varphi^{2}\right)
\end{aligned}
$$

where $c_{4}$ is a constant depending on $n$. We see that the left hand side of the last inequality is a quadratic polynomial in $(\varphi G)$; then using the quadratic formula and the elementary inequality $\sqrt{1+a^{2}+p^{2}} \leq 1+a+p$ yields

$$
\begin{aligned}
\varphi G & \leq \frac{\alpha n p}{4}+\frac{\alpha n p}{4 \rho^{2}} c_{4} t\left(\frac{p \alpha^{3}}{(\alpha-1)}+\rho \sqrt{\rho_{1}}+\frac{\rho^{2}}{\tau}+\rho^{2} \widehat{\rho}\right)+\frac{\alpha n p}{4}\left(\frac{2 \alpha \rho_{1}}{\alpha-1} t+2 \widehat{\rho}^{\frac{1}{2}} \varphi t \sqrt{\frac{q}{p}}\right) \\
& =\frac{\alpha n p}{4}+\frac{\alpha n p}{4 \rho^{2}} c_{4} t\left(\frac{p \alpha^{3}}{(\alpha-1)}+\frac{\rho^{2}}{\tau}+\rho^{2} \widehat{\rho}\right)+\frac{\alpha^{2} n p \rho_{1}}{2(\alpha-1)} t+\frac{\alpha n}{2} t \widehat{\rho}^{\frac{1}{2}} \sqrt{p q} .
\end{aligned}
$$


Recall that we picked up $\varphi(x, t)$ such that $0 \leq \varphi \leq 1$ and, in particular, $\varphi(x, t)=$ 1 in $\mathcal{Q}_{2 \rho, T}$, and since $\left(x_{0}, t_{0}\right)$ is a maximum point for $(\varphi G)$ in $\mathcal{Q}_{2 \rho, T}$, we have $G(x, \tau)=(\varphi G)(x, \tau) \leq(\varphi G)\left(x_{0}, t_{0}\right)$. Hence

$$
\frac{1}{t} G(x, t) \leq \frac{\alpha n p}{4 t}+\frac{\alpha n}{4 \rho^{2}} c_{4}\left(\frac{\alpha p}{(\alpha-1)}+\frac{\rho^{2}}{\tau}+\rho^{2} \widehat{\rho}\right)+\frac{\alpha^{2} n p}{2(\alpha-1)} \rho_{1}+\frac{\alpha n}{2} \widehat{\rho}^{\frac{1}{2}} \sqrt{p q},
$$

for all $x \in M$ such that $d\left(x, x_{0}, \tau\right)<\rho$ and $\tau \in(0, T]$, and this completes the proof.

Let $(M, g)$ be a screen integrable globally null manifold. Let $\left(M^{\prime}, g^{\prime}\right)$ be an $n$-dimensional compact (or noncompact without boundary) leaf of $S(T M)$ with bounded Ricci curvature. Using Lemma 5.1 and local gradient in Theorem 5.2 we now present global estimates for the positive solutions to the heat equation when the metric $g^{\prime}$ evolves by the degenerate Ricci-type flow.

Theorem 5.3. Let $(M, g)$ be a screen integrable globally null manifold and let $\left(M^{\prime}, g^{\prime}(t)\right)$ be a complete leaf of $S(T M)$. Assume that $g^{\prime}(t)$ solves the degenerate Ricci-type flow equation such that its associated Ricci curvature is bounded for all $(x, t) \in M^{\prime} \times(0, T]$. Let $u=u(x, t)>0$ be any positive solution to the heat equation 5.2). Then we have, for $-\rho_{1} g^{\prime}(x, t) \leq \operatorname{Ric}^{\prime}(x, t) \leq \rho_{2} g^{\prime}(x, t)$ and $\alpha>1$ with $\frac{1}{p}+\frac{1}{q}=\frac{1}{\alpha}$,

$$
\frac{\|\bar{\nabla} u\|^{2}}{u^{2}}-\alpha \frac{u_{t}}{u} \leq \frac{\alpha n p}{4 t}+\frac{\alpha^{2} n p}{2(\alpha-1)} \rho_{1}+\frac{\alpha n}{2}\left(\rho_{1}+\rho_{2}\right) \sqrt{p q},
$$

for all $(x, t) \in M^{\prime} \times(0, T]$. Moreover, if we assume that $M^{\prime}$ has non-negative Ricci curvature in the case of a compact manifold, i.e., for $0 \leq \operatorname{Ric}^{\prime}(x, t) \leq \rho g^{\prime}(x, t)$, we have

$$
\frac{\|\bar{\nabla} u\|^{2}}{u^{2}}-\alpha \frac{u_{t}}{u} \leq \frac{\alpha n p}{4 t}+\frac{\alpha n}{2} \rho \sqrt{p q}
$$

for all $(x, t) \in M^{\prime} \times(0, T]$ and $\alpha \geq 1$ with $\frac{1}{p}+\frac{1}{q}=\frac{1}{\alpha}$.

Proof. Set $f=\ln u$ and allow $G$ to remain as before. At the maximum point $\left(x_{0}, t_{0}\right) \in M^{\prime} \times(0, T]$, we will show that a new function

$$
\widetilde{G}=G-\frac{\alpha^{2} n p}{2(\alpha-1)} t \rho_{1}-\frac{\alpha n}{2} t\left(\rho_{1}+\rho_{2}\right) \sqrt{p q}
$$

satisfies the inequality

$$
\widetilde{G} \leq \frac{\alpha n p}{4}+\frac{\alpha^{2} n p}{2(\alpha-1)} t \rho_{1}+\frac{\alpha n}{2} t\left(\rho_{1}+\rho_{2}\right) \sqrt{p q} .
$$

It suffices to prove that $\widetilde{G} \leq \alpha n p$ for any $\left(x_{0}, t_{0}\right) \in M^{\prime} \times(0, T]$. We now show this by contradiction. Suppose that $\widetilde{G}>\alpha n p$ and $\widetilde{G}$ has its maximum at the point $\left(x_{0}, t_{0}\right)$; then we know that $\bar{\nabla} \widetilde{G}\left(x_{0}, t_{0}\right)=0, \Delta \widetilde{G}\left(x_{0}, t_{0}\right) \leq 0, \frac{\partial}{\partial t} \widetilde{G}\left(x_{0}, t_{0}\right) \geq 0$, and 
$\left(\underline{\Delta}-\partial_{t}\right) \widetilde{G}\left(x_{0}, t_{0}\right) \leq 0$. Then, by Lemma 5.1 , we have $0 \geq\left(\underline{\Delta}-\partial_{t}\right) \widetilde{G} \geq\left(\underline{\Delta}-\partial_{t}\right) G$. Note that

$$
\left(\|\bar{\nabla} f\|^{2}-\partial_{t} f\right)^{2}=\frac{1}{\alpha^{2}}\left(\frac{G}{t_{0}}\right)^{2}+\frac{2(\alpha-1)}{\alpha^{2}}\|\bar{\nabla} f\|^{2}\left(\frac{G}{t_{0}}\right)+\frac{(\alpha-1)^{2}}{\alpha}\|\bar{\nabla} f\|^{4} .
$$

By the expression 4.8,

$$
\begin{aligned}
0 \geq\left(\underline{\Delta}-\partial_{t}\right) G \geq & -2 \bar{\nabla} f \bar{\nabla} G+\frac{2 \alpha}{n p} t_{0}\left(\|\bar{\nabla} f\|^{2}-\partial_{t} f\right)^{2}-\left(\|\bar{\nabla} f\|^{2}-\alpha \partial_{t} f\right) \\
& -2 \alpha t_{0} \rho_{1}\|\bar{\nabla} f\|^{2}-\frac{\alpha n q}{2} t_{0}\left(\rho_{1}+\rho_{2}\right)^{2} .
\end{aligned}
$$

Following the calculation in Theorem 5.2 , we obtain

$$
\frac{G}{t_{0}} \leq \frac{\alpha n p}{4 t_{0}}+\frac{\alpha^{2} n p}{2(\alpha-1)} \rho_{1}+\frac{\alpha n}{2}\left(\rho_{1}+\rho_{2}\right)^{2} \sqrt{p q},
$$

after letting $\rho$ tend to $\infty$. Consequently, we have the inequality

$$
0 \geq \frac{2 t_{0}}{\alpha n p}\left(\frac{G}{t_{0}}\right)^{2}-\frac{G}{t_{0}}-\left(\frac{\alpha^{2} n p}{2(\alpha-1)} \rho_{1}+\frac{\alpha n}{2}\left(\rho_{1}+\rho_{2}\right)^{2} \sqrt{p q}\right)
$$

resulting in a quadratic inequality, and since from (5.11)

$$
\frac{G}{t_{0}}=\frac{\widetilde{G}}{t_{0}}+\frac{\alpha^{2} n p}{2(\alpha-1)} \rho_{1}+\frac{\alpha n}{2}\left(\rho_{1}+\rho_{2}\right)^{2} \sqrt{p q},
$$

we obtain

$$
\frac{2 t_{0}}{\alpha n p}\left(\frac{G}{t_{0}}\right)^{2}-\frac{G}{t_{0}}-\frac{\alpha^{2} n p}{2(\alpha-1)} \rho_{1} \leq 0 .
$$

Using the quadratic formula, we have

$$
\frac{G}{t_{0}} \leq \frac{\alpha n p}{4 t_{0}}\left\{1+\sqrt{1+\frac{4 \alpha n p}{(\alpha-1)}} \rho_{1}\right\}
$$

which obviously implies that $\widetilde{G} \leq \alpha n p$, a contradiction with the assumption 5.12 . By definition of $\widetilde{G}$, we therefore have

$$
\frac{G}{t_{0}}=\frac{\|\bar{\nabla} u\|^{2}}{u^{2}}-\frac{u_{t}}{u} \leq \frac{\widetilde{G}}{t_{0}}+\frac{\alpha^{2} n p}{2(\alpha-1)} \rho_{1}+\frac{\alpha n}{2}\left(\rho_{1}+\rho_{2}\right) \sqrt{p q} .
$$

The desired estimate follows since $t_{0}$ was arbitrarily chosen.

The case $\alpha=1$ leads to the following result, in which we choose $p=q=2$.

Theorem 5.4. Let $(M, g)$ be a compact screen integrable globally null manifold and let $\left(M^{\prime}, g^{\prime}(t)\right)$, with $t \in(0, T]$, be a complete leaf of $S(T M)$. Assume that $g^{\prime}(t)$ solves the degenerate Ricci-type flow equation 5.2 , and also that $0 \leq \operatorname{Ric}^{\prime}(x, t) \leq$ $\rho g^{\prime}(x, t)$ for a positive constant $\rho>0$ and for all $(x, t) \in M^{\prime} \times(0, T]$. Let $u=$ $u(x, t)>0$ be any positive solution to the heat equation (5.2). Then, the estimate

$$
\frac{\|\bar{\nabla} u\|^{2}}{u^{2}}-\frac{u_{t}}{u} \leq \frac{n}{2 t}+n \rho
$$


holds, for all $(x, t) \in M^{\prime} \times(0, T]$.

Proof. As before, set $f=\ln u$ and $G=t\left(\|\bar{\nabla} f\|^{2}-\partial_{t} f\right)$. Fix $\tau \in(0, T]$ and choose a point $\left(x_{0}, t_{0}\right) \in M^{\prime} \times(0, \tau]$ where $G_{1}$ attains its maximum on $M^{\prime} \times(0, \tau]$. Now we can show that

$$
G\left(x_{0}, t_{0}\right) \leq t_{0} n \rho+\frac{n}{2} .
$$

If $t_{0}=0$, then $G\left(x_{0}, t_{0}\right)=0$, for every $x \in M$. From this, we can get estimate 5.13. Next, we can assume that $t_{0}>0$. Lemma 5.1 and the conditions on the Ricci curvature of $M$ lead to the inequality

$$
\left(\underline{\Delta}-\partial_{t}\right) G \geq-2 \bar{g}(\bar{\nabla} f, \bar{\nabla} G)+\frac{2 p}{n} \frac{G^{2}}{t_{0}}-\frac{G}{t_{0}}-\frac{t_{0} n}{2(1-p)} \rho^{2} .
$$

Recall that $G$ attains its maximum at $\left(x_{0}, t_{0}\right)[3$. This gives

$$
\Delta G\left(x_{0}, t_{0}\right) \leq 0, \quad \frac{\partial}{\partial t} G\left(x_{0}, t_{0}\right) \geq 0, \quad \bar{\nabla} G\left(x_{0}, t_{0}\right)=0 .
$$

Then, the estimate

$$
\frac{2 p}{n} \frac{G^{2}}{t_{0}}-\frac{G}{t_{0}}-\frac{t_{0} n}{2(1-p)} \rho^{2} \leq 0
$$

holds at $\left(x_{0}, t_{0}\right)$, and the quadratic formula yields

$$
G\left(x_{0}, t_{0}\right) \leq \frac{n}{4 p}\left\{1+\sqrt{1+\frac{4 p t_{0}^{2}}{(1-p)}} \rho^{2}\right\} .
$$

Now recall that $\left(x_{0}, t_{0}\right)$ is a maximum point for $G$ on $M^{\prime} \times(0, \tau]$; from this fact, we can get that

$$
G(x, \tau) \leq G\left(x_{0}, t_{0}\right) \leq t_{0} \rho n+\frac{n}{2} \leq \tau \rho n+\frac{n}{2},
$$

for all $x \in M$. Therefore, the estimate

$$
\frac{\|\bar{\nabla} u\|^{2}}{u^{2}}-\frac{u_{t}}{u} \leq \rho n+\frac{n}{2 \tau}
$$

holds at $(x, \tau)$, for any $\tau \in(0, T]$.

Corollary 5.5. Let $(M, g)$ be a screen integrable globally null manifold and let $\left(M^{\prime}, g^{\prime}\right)$ be a complete leaf of $S(T M)$. Assume that $g^{\prime}(t)$ solves the degenerate Ricci-type flow equation (5.1) such that its Ricci curvature is bounded for all $(x, t) \in$ $M^{\prime} \times(0, T]$. Let $u=u(x, t)>0$ be any positive solution to the heat equation (5.2), and $0<u \leq A$. Then, for $-\rho_{1} g^{\prime}(x, t) \leq \operatorname{Ric}^{\prime}(x, t) \leq \rho_{2} g^{\prime}(x, t)$, there exists an absolute constant $C$ such that

$$
t\|\bar{\nabla} u\|^{2} \leq C A\left(1+\rho_{1} T\right) \quad \text { in } M^{\prime} \times(0, T] .
$$

Also, we have the following. 
Corollary 5.6. Let $(M, g)$ be a screen integrable globally null manifold and let $\left(M^{\prime}, g^{\prime}\right)$ be a complete leaf of $S(T M)$. Assume that $\left(M^{\prime}, g^{\prime}(t)\right)$ is a complete solution of the degenerate Ricci-type flow (5.1) such that the Ricci curvature satisfies

$$
-\rho_{1} g^{\prime}(x, t) \leq \operatorname{Ric}^{\prime}(x, t) \leq \rho_{2} g^{\prime}(x, t),
$$

for all $(x, t) \in M^{\prime} \times(0, T]$. Let $u=u(x, t)$ be any positive solution to the heat equation 5.2. Then, for $\alpha>1$ with $\frac{1}{p}+\frac{1}{q}=\frac{1}{\alpha}$, we have

$$
\frac{\|\bar{\nabla} u\|^{2}}{u^{2}}-\frac{u_{t}}{u} \leq \frac{\alpha n p}{4 t}+c(n) \alpha^{2}\left(\rho_{1}+\rho_{2}\right),
$$

for all $(x, t) \in M^{\prime} \times(0, T]$ and an absolute constant $c(n)$ depending only on $n$.

\section{REFERENCES}

[1] A. Abolarinwa, Gradient estimates for heat-type equations on evolving manifolds, J. Nonlinear Evol. Equ. Appl. 2015, 1-19. MR 3326817

[2] B. Andrews and C. Hopper, The Ricci Flow in Riemannian Geometry, Lecture Notes in Mathematics, 2011, Springer, Heidelberg, 2011. MR 2760593

[3] M. Bailesteanu, X. Cao and A. Pulemotov, Gradient estimates for the heat equation under the Ricci flow, J. Funct. Anal. 258 (2010), no. 10, 3517-3542. MR 2601627

[4] E. Calabi, An extension of E. Hopf's maximum principle with an application to Riemannian geometry, Duke Math. J. 25 (1958), 45-56. MR 0092069

[5] J. Cheeger and S. T. Yau, A lower bound for the heat kernel, Comm. Pure Appl. Math. 34 (1981), no. 4, 465-480. MR 0615626

[6] B. Chow et al., The Ricci Flow: Techniques and Applications. Part IV. Long-time solutions and related topics, Mathematical Surveys and Monographs, 206, American Mathematical Society, Providence, RI, 2015. MR 3409114

[7] K. L. Duggal and A. Bejancu, Lightlike Submanifolds of Semi-Riemannian Manifolds and Applications, Mathematics and its Applications, 364, Kluwer, Dordrecht, 1996. MR 1383318.

[8] K. L. Duggal, Warped product of lightlike manifolds, Nonlinear Anal. 47 (2001), no. 5, 3061-3072. MR 1979204.

[9] K. L. Duggal and D. H. Jin, Geometry of null curves, Math. J. Toyama Univ. 22 (1999), 95-120. MR 1744499

[10] K. L. Duggal and B. Sahin, Differential Geometry of Lightlike Submanifolds, Frontiers in Mathematics, Birkhäuser Verlag, Basel, 2010. MR 2598375

[11] K. Ecker et al., Local monotonicity and mean value formulas for evolving Riemannian manifolds, J. Reine Angew. Math. 616 (2008), 89-130. MR 2369488

[12] R. S. Hamilton, Three-manifolds with positive Ricci curvature, J. Differential Geometry 17 (1982), no. 2, 255-306. MR 0664497

[13] M. Kossowski, The intrinsic conformal structure and Gauss map of a light-like hypersurface in Minkowski space, Trans. Amer. Math. Soc. 316 (1989), no. 1, 369-383. MR 0938920

[14] D. N. Kupeli, Singular Semi-Riemannian Geometry, Mathematics and its Applications, 366, Kluwer, Dordrecht, 1996. MR 1392222

[15] P. Li and S.-T. Yau, On the parabolic kernel of the Schrödinger operator, Acta Math. 156 (1986), no. 3-4, 153-201. MR 0834612

[16] F. Massamba, Totally contact umbilical lightlike hypersurfaces of indefinite Sasakian manifolds, Kodai Math. J. 31 (2008), no. 3, 338-358. MR 2475274 
[17] F. Massamba, Screen conformal invariant lightlike hypersurfaces of indefinite Sasakian space forms, Afr. Diaspora J. Math. 14 (2012), no. 2, 22-37. MR 3093232

[18] F. Massamba, On semi-parallel lightlike hypersurfaces of indefinite Kenmotsu manifolds, J. Geom. 95 (2009), no. 1-2, 73-89. MR 2595664

[19] F. Massamba, On lightlike geometry in indefinite Kenmotsu manifolds, Math. Slovaca 62 (2012), no. 2, 315-344. MR 2891725.

[20] F. Massamba and S. Ssekajja, A new approach to totally umbilical null submanifolds, Novi Sad J. Math. 47 (2017), no. 2, 63-76. MR 3762652.

[21] B. O'Neill, Semi-Riemannian Geometry, Pure and Applied Mathematics, 103, Academic Press, New York, 1983. MR 0719023

[22] G. Perelman, The entropy formula for the Ricci flow and its geometric applications, preprint, 2002. arXiv:math/0211159 [math.DG].

[23] R. Schoen and S.-T. Yau, Lectures on Differential Geometry, Conference Proceedings and Lecture Notes in Geometry and Topology, I, International Press, Cambridge, MA, 1994. MR 1333601

\section{H. A. Hamed}

School of Mathematics, Statistics and Computer Science, University of KwaZulu-Natal, Private Bag X01, Scottsville 3209, South Africa

wdhamed82@gmail.com

F. Massamba ${ }^{凶}$

School of Mathematics, Statistics and Computer Science, University of KwaZulu-Natal, Private Bag X01, Scottsville 3209, South Africa

massfort@yahoo.fr, Massamba@ukzn.ac.za

S. Ssekajja

School of Mathematics, Statistics and Computer Science, University of KwaZulu-Natal, Private Bag X01, Scottsville 3209, South Africa

ssekajja.samuel.buwaga@aims-senegal.org

Received: December 24, 2019

Accepted: April 21, 2020 